\title{
Forward instrumentation for ILC detectors
}

This article has been downloaded from IOPscience. Please scroll down to see the full text article.

2010 JINST 5 P12002

(http://iopscience.iop.org/1748-0221/5/12/P12002)

View the table of contents for this issue, or go to the journal homepage for more

Download details:

IP Address: 141.34.3.101

The article was downloaded on 15/02/2011 at $14: 42$

Please note that terms and conditions apply. 


\section{Forward instrumentation for ILC detectors}
H. Abramowicz, ${ }^{a}$ A. Abusleme, ${ }^{b}$ K. Afanaciev,${ }^{c}$ J. Aguilar, ${ }^{d}$ P. Ambalathankandy, ${ }^{d}$
P. Bambade, ${ }^{e}$ M. Bergholz, ${ }^{f, 1}$ I. Bozovic-Jelisavcic, ${ }^{g}$ E. Castro, ${ }^{f}$ G. Chelkov, ${ }^{h}$
C. Coca, ${ }^{i}$ W. Daniluk, ${ }^{j}$ A. Dragone,${ }^{k}$ L. Dumitru, ${ }^{i}$ K. Elsener ${ }^{l}$ I. Emeliantchik, ${ }^{c}$
T. Fiutowski, ${ }^{d}$ M. Gostkin, ${ }^{h}$ C. Grah, ${ }^{f, 2}$ G. Grzelak,,${ }^{j, 3}$ G. Haller,${ }^{k}$ H. Henschel, ${ }^{f}$
A. Ignatenko, ${ }^{c, 4}$ M. Idzik, ${ }^{d}$ K. Ito, ${ }^{m}$ T. Jovin, ${ }^{g}$ E. Kielar, ${ }^{j}$ J. Kotula, ${ }^{j}$ Z. Krumstein, ${ }^{h}$
S. Kulis, ${ }^{d}$ W. Lange, ${ }^{f}$ W. Lohmann,,${ }^{f, 1,5}$ A. Levy, ${ }^{a}$ A. Moszczynski, ${ }^{j}$ U. Nauenberg, ${ }^{n}$
O. Novgorodova, ${ }^{f, 1}$ M. Ohlerich,,${ }^{f, 1}$ M. Orlandea, ${ }^{i}$ G. Oleinik, ${ }^{n}$ K. Oliwa, ${ }^{j}$

A. Olshevski, ${ }^{h}$ M. Pandurovic, ${ }^{g}$ B. Pawlik, ${ }^{j}$ D. Przyborowski, ${ }^{d}$ Y. Sato, ${ }^{m}$ I. Sadeh, ${ }^{a}$

A. Sailer, ${ }^{l}$ R. Schmidt, ${ }^{f, 1}$ B. Schumm, ${ }^{o}$ S. Schuwalow, ${ }^{f}$ I. Smiljanic, ${ }^{g}$ K. Swientek, ${ }^{d}$

Y. Takubo, ${ }^{m}$ E. Teodorescu, ${ }^{i}$ W. Wierba, ${ }^{j}$ H. Yamamoto, ${ }^{m}$ L. Zawiejski ${ }^{j}$ and J. Zhang ${ }^{p}$

${ }^{a}$ Tel Aviv University, Tel Aviv, Israel

${ }^{b}$ Stanford University, Stanford, U.S.A.

${ }^{c}$ NCPHEP, Minsk, Belarus

${ }^{d}$ AGH University of Science \& Technology, Cracow, Poland

${ }^{e}$ Laboratoire de 1 Accelerateur Lineaire, Orsay, France

${ }^{f}$ DESY, Zeuthen, Germany

${ }^{g}$ Vinca Institute of Nuclear Sciences, University of Belgrade, Serbia

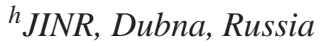

${ }^{i}$ IFIN-HH, Bucharest, Romania

${ }^{j}$ INP PAN, Cracow, Poland

${ }^{k}$ SLAC, Menlo Park, U.S.A.

${ }^{l}$ CERN, Geneva, Switzerland

${ }^{m}$ Tohoku University, Sendai, Japan

${ }^{n}$ University of Colorado, Boulder, U.S.A.

${ }^{o}$ UC California, Santa Cruz, U.S.A.

${ }^{p}$ ANL, Argonne, U.S.A.

E-mail: Wolfgang. Lohmann@desy.de

\footnotetext{
${ }^{1}$ Also at Brandenburg University of Technology, Cottbus, Germany

${ }^{2}$ Now at BTO Consulting AG, Berlin, Germany

${ }^{3}$ Also at University of Warsaw, Poland

${ }^{4}$ Now at DESY, Hamburg, Germany

${ }^{5}$ Corresponding author.
} 
ABSTRACT: Two special calorimeters are foreseen for the instrumentation of the very forward region of the ILC detector, a luminometer designed to measure the rate of low angle Bhabha scattering events with a precision better than $10^{-3}$ and a low polar angle calorimeter, adjacent to the beam-pipe. The latter will be hit by a large amount of beamstrahlung remnants. The amount and shape of these depositions will allow a fast luminosity estimate and the determination of beam parameters. The sensors of this calorimeter must be radiation hard. Both devices will improve the hermeticity of the detector in the search for new particles. Finely segmented and very compact calorimeters will match the requirements. Due to the high occupancy fast front-end electronics is needed. The design of the calorimeters developed and optimised with Monte Carlo simulations is presented. Sensors and readout electronics ASICs have been designed and prototypes are available. Results on the performance of these major components are summarised.

KEYWORDS: Si microstrip and pad detectors; Radiation-hard detectors; Calorimeter methods; Detector modelling and simulations I (interaction of radiation with matter, interaction of photons with matter, interaction of hadrons with matter, etc)

ARXIV EPRINT: 1009.2433 


\section{Contents}

1 Introduction and challenges $\quad 2$

2 Design of the very forward region 3

2.1 LumiCal simulation studies 3

$\begin{array}{ll}2.2 & \text { BeamCal simulation studies }\end{array}$

$\begin{array}{lll}2.3 & \text { Pair monitor simulations } & 7\end{array}$

3 Mechanical concepts 9

4 Systematic effects in the luminosity measurement $\quad 11$

4.1 Pinch effect and beamstrahlung 11

$\begin{array}{ll}\text { 4.2 Background from four-fermion production } & 12\end{array}$

4.3 Effects of a bias in the energy resolution and the energy scale 13

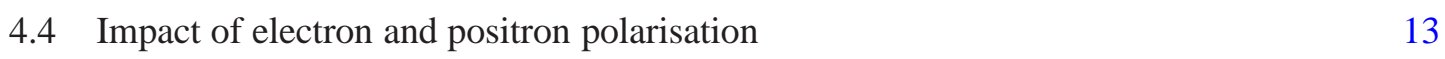

$\begin{array}{ll}\text { 4.5 Summary of systematic uncertainties } & 13\end{array}$

5 Sensor development $\quad 14$

$\begin{array}{lll}5.1 & \text { Sensors for BeamCal } & 14\end{array}$

$\begin{array}{lll}5.1 .1 & \text { GaAs sensors } & 15\end{array}$

$\begin{array}{ll}\text { 5.1.2 CVD diamond sensors } & 15\end{array}$

$\begin{array}{ll}5.2 \text { Sensors for LumiCal } & 16\end{array}$

6 ASIC developments 17

$\begin{array}{lll}6.1 & \text { LumiCal readout } & 17\end{array}$

$\begin{array}{lll}\text { 6.1.1 Front-end electronics design } & 18\end{array}$

6.1.2 Front-end electronics measurements 19

$\begin{array}{lll}6.1 .3 & \text { ADC design } & 20\end{array}$

6.1.4 ADC performance measurements 20

$\begin{array}{ll}6.2 \text { BeamCal readout } & 20\end{array}$

6.2.1 Circuit implementation 22

$\begin{array}{lll}\text { 6.2.2 Test results } & 23\end{array}$

6.3 Pair monitor readout 24

$\begin{array}{lll}7 & \text { Summary } & 25\end{array}$ 


\section{Introduction and challenges}

A high energy $\mathrm{e}^{+} \mathrm{e}^{-}$linear collider is considered to be the future research facility complementary to the LHC collider. Whereas LHC has a higher potential for discoveries, an $\mathrm{e}^{+} \mathrm{e}^{-}$collider will allow precision measurements to explore in detail the mechanism of electroweak symmetry breaking and the properties of the physics beyond the Standard Model, should it be found at the LHC. Two concepts of an $\mathrm{e}^{+} \mathrm{e}^{-}$linear collider are presently considered, the ILC [1] and CLIC [2]. For the ILC, with superconducting cavities, an engineering design report will be issued in 2012. The centre-of-mass energy will be $500 \mathrm{GeV}$, with the possibility of an upgrade to $1 \mathrm{TeV}$. CLIC is based on conventional cavities. A conceptional design report is foreseen in 2011. CLIC will allow to collide electrons and positrons up to energies of $3 \mathrm{TeV}$.

An R\&D program is ongoing to develop the technologies for detectors for precision measurements in this new energy domain. Letters of Intent have been submitted for detectors at the ILC in 2009. Two detectors, the ILD [3] and the SiD [4], are reviewed and validated. In both detectors two specialised calorimeters are foreseen in the very forward region, LumiCal for the precise measurement of the luminosity and BeamCal for a fast estimate of the luminosity and for the control of beam parameters [5]. Both will also improve the hermeticity of the detector. To support beam-tuning an additional pair-monitor will be positioned just in front of BeamCal.

With LumiCal the luminosity will be measured using Bhabha scattering, $\mathrm{e}^{+} \mathrm{e}^{-} \rightarrow \mathrm{e}^{+} \mathrm{e}^{-}(\gamma)$, as a gauge process. To match the physics benchmarks, an accuracy of better than $10^{-3}$ is needed at a centre-of-mass energy of $500 \mathrm{GeV}$ [3]. For the GigaZ option, where the ILC will be operated for precision measurements at centre-of-mass energies around the $\mathrm{Z}$ boson, an accuracy of $10^{-4}$ would be required [6]. To reach these accuracies, a precision device is needed, with particularly challenging requirements on the mechanics and position control.

BeamCal is positioned just outside the beam-pipe. At ILC energies we have to tackle here a new phenomenon - the beamstrahlung. When electron and positron bunches collide, the particles are accelerated in the magnetic field of the bunches towards the bunch centre. This so called pinch effect enhances the luminosity. However, electrons and positrons may radiate photons. A fraction of these photons converts in the Coulomb field of the bunch particles creating low energy $\mathrm{e}^{+} \mathrm{e}^{-}$ pairs. A large amount of these pairs will deposit their energy after each bunch crossing in BeamCal. These depositions, useful for a bunch-by-bunch luminosity estimate and the determination of beam parameters [7], will lead, however, to a radiation dose of about one MGy per year in the sensors at lower polar angles. Hence radiation hard sensors are needed to instrument BeamCal. BeamCal is supplemented by a pair monitor, consisting of a layer of pixel sensors positioned just in front of it to measure the density of beamstrahlung pairs and give additional information for the beam parameter determination.

All detectors in the very forward region have to tackle relatively high occupancy, requiring special front-end electronics.

A small Molière radius is of importance for both calorimeters. It ensures high energy electron veto capability for BeamCal even at small polar angles. This is essential to suppress background in searches for new particles for which the signature consists of large missing energy and momentum. In LumiCal the precise reconstruction of electron, positron and photon showers in Bhabha events is facilitated. Both calorimeters also shield the inner tracking detectors from back-scattered particles 


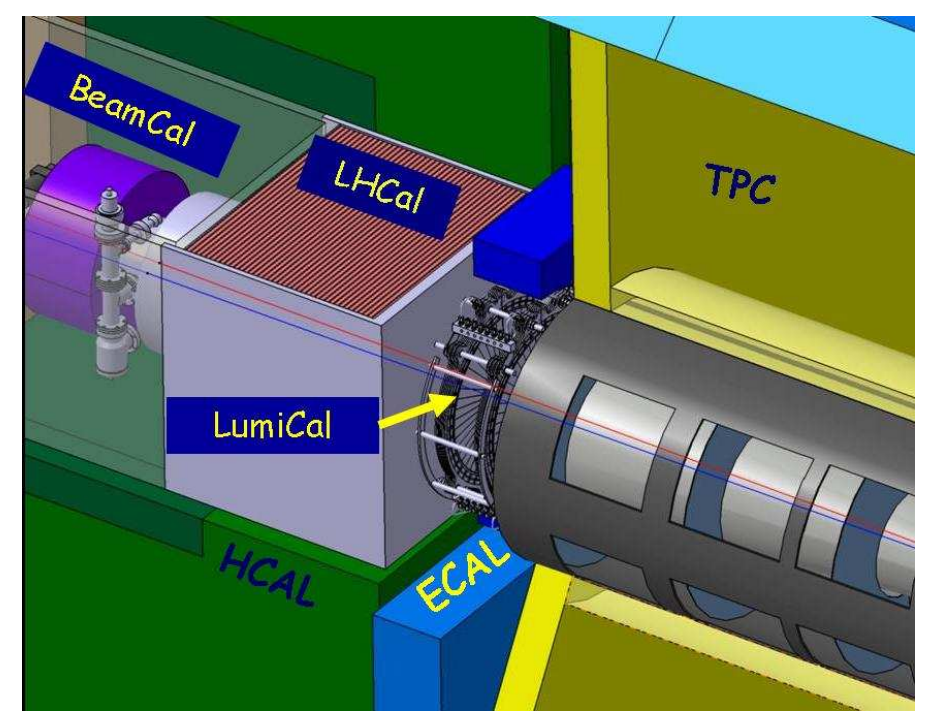

Figure 1: The very forward region of the ILD detector. LumiCal, BeamCal and LHCal are carried by the support tube for the final focusing quadrupole and the beam-pipe. LHCal extends the coverage of the hadron calorimeter to the polar angle range of LumiCal. TPC denotes the central track chamber, ECAL the electromagnetic and HCAL the hadron calorimeter.

induced by beamstrahlung pairs hitting the downstream beam-pipe and magnets.

\section{Design of the very forward region}

A sketch of the very forward region of the ILD detector [3] is shown in figure 1. LumiCal and BeamCal are designed as cylindrical sensor-tungsten sandwich electromagnetic calorimeters. Both consist of 30 absorber disks of $3.5 \mathrm{~mm}$ thickness, each corresponding to one radiation length, interspersed with sensor layers. Each sensor layer is segmented radially and azimuthally into pads. Front-end ASICs are positioned at the outer radius of the calorimeters. LumiCal is positioned in a circular hole of the end-cap electromagnetic calorimeter ECAL. BeamCal is placed just in front of the final focus quadrupole. BeamCal covers polar angles between 5 and $40 \mathrm{mrad}$ and LumiCal between 31 and $77 \mathrm{mrad}$.

Colliding beams enter the interaction point, IP, with a crossing angle of $14 \mathrm{mrad}$. Both calorimeters are centred around the outgoing beam. In the design of BeamCal a hole for the incoming beam-pipe is foreseen.

\subsection{LumiCal simulation studies}

The differential cross section of Bhabha scattering, $\frac{d \sigma_{B}}{d \theta}$, can be calculated precisely from theory [8]. In leading order it reads,

$$
\frac{d \sigma_{\mathrm{B}}}{d \theta}=\frac{2 \pi \alpha_{\mathrm{em}}^{2}}{s} \frac{\sin \theta}{\sin ^{4}(\theta / 2)} \approx \frac{32 \pi \alpha_{\mathrm{em}}^{2}}{s} \frac{1}{\theta^{3}},
$$

where $\theta$ is the polar angle of the scattered electron with respect to the beam. The approximation holds at small $\theta$. 


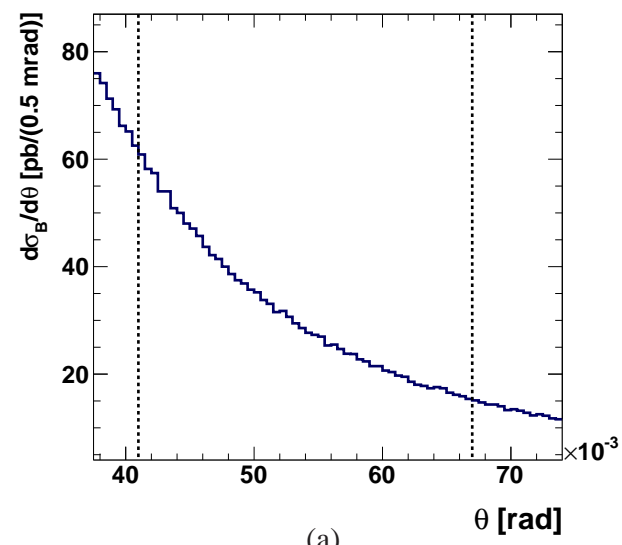

(a)

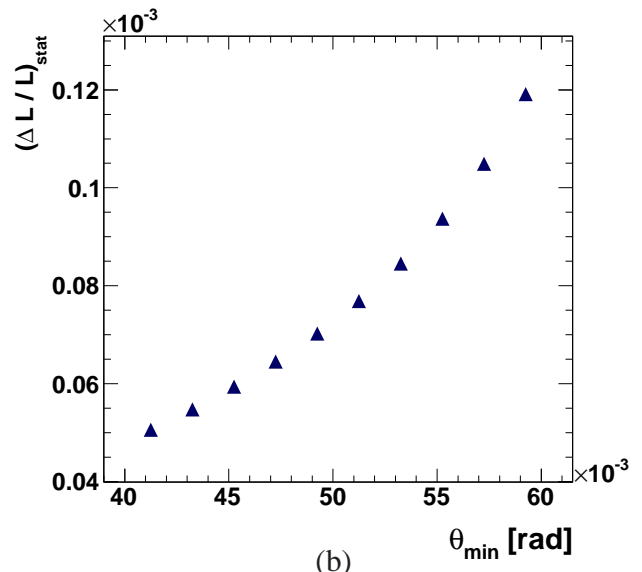

(b)

Figure 2: (a) Dependence of $d \sigma_{\mathrm{B}} / d \theta$, the differential Bhabha cross-section, on the polar angle, $\theta$, at $\sqrt{s}=500 \mathrm{GeV}$. The dashed lines mark the fiducial volume of LumiCal, $41<\theta<67 \mathrm{mrad}$, which is defined in eq. (2.5) later in this section. (b) Dependence of the statistical uncertainty in counting the number of Bhabha events, $(\Delta \mathrm{L} / \mathrm{L})_{\text {stat }}$, on the minimal polar angle of the fiducial volume, $\theta_{\min }$, while the upper limit is kept at $67 \mathrm{mrad}$. An integrated luminosity of $500 \mathrm{fb}^{-1}$ is assumed.

For a given rate of Bhabha events, $\mathrm{N}_{\mathrm{B}}$, determined in a certain $\theta$-range, the luminosity, $\mathrm{L}$, is obtained as

$$
L=\frac{N_{B}}{\sigma_{B}},
$$

where $\sigma_{\mathrm{B}}$ is the integral of the differential cross section, eq. (2.1), over the considered $\theta$ range. Because of the steep $\theta$ dependence of the cross section, as illustrated in figure $2 \mathrm{a}$, the most critical quantity to control when counting Bhabha events is the inner acceptance radius of the calorimeter, defined as the lower cut in the polar angle, $\theta_{\min }$. Hence a very precise $\theta$ measurement is needed. Furthermore, the $\theta$-range must be chosen such that the number of Bhabha events measured provides the required relative statistical uncertainty of $10^{-3}$. By choosing the lower bound of the polar angle between 40 and $60 \mathrm{mrad}$ the latter requirement can be easily reached as illustrated in figure $2 \mathrm{~b}$. Here a Bhabha event sample generated with the BHWIDE generator [9] was used. The generated sample corresponds to an integrated luminosity of $500 \mathrm{fb}^{-1}$, as expected in one year of running the collider at nominal luminosity.

Electromagnetic showers are simulated in LumiCal using the GEANT4 [10] based package Mokka [11]. Sensors consist of $300 \mu \mathrm{m}$ thick silicon sectors covering an azimuthal angle of $30^{\circ}$. The depositions in each sensor pad are recorded, and a reconstruction of the shower is performed. The position of an electromagnetic shower in LumiCal is reconstructed by performing a weighted average over the energy deposits in individual pads. The weight, $\mathscr{W}_{\mathrm{i}}$, of a given detector pad $\mathrm{i}$ is determined by logarithmic weighting [12], for which $\mathscr{W}_{\mathrm{i}}=\max \left\{0, \mathscr{C}+\ln \left(\mathrm{E}_{\mathrm{i}} / \mathrm{E}_{\text {tot }}\right)\right\}$. Here $\mathrm{E}_{\mathrm{i}}$ refers to the individual pad energy, $\mathrm{E}_{\text {tot }}$ is the total energy in all pads, and $\mathscr{C}$ is a constant. In this way, only pads which contain a sufficient fraction of the shower energy contribute to the reconstruction. The polar angle resolution, $\sigma_{\theta}$, and a polar angle measurement bias, $\Delta \theta$, are defined as the Gaussian width and the central value of the difference between the reconstructed and the generated polar angles. There is an optimal value for $\mathscr{C}$, for which $\sigma_{\theta}$ is minimal $[13,14]$. 


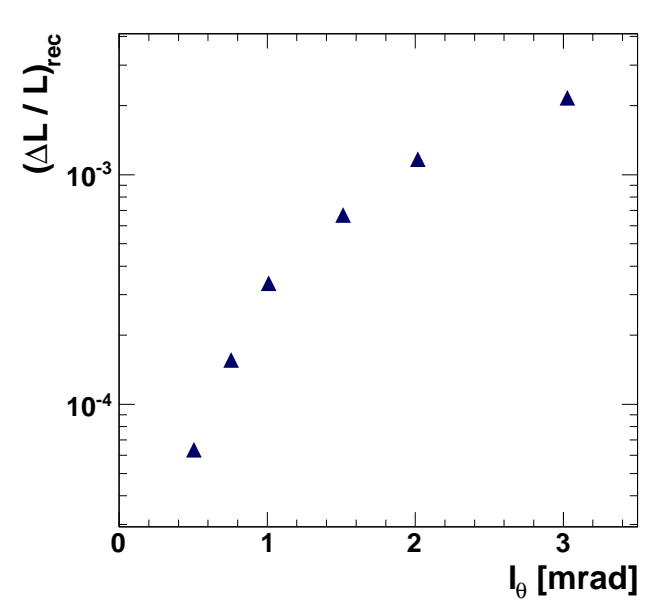

(a)

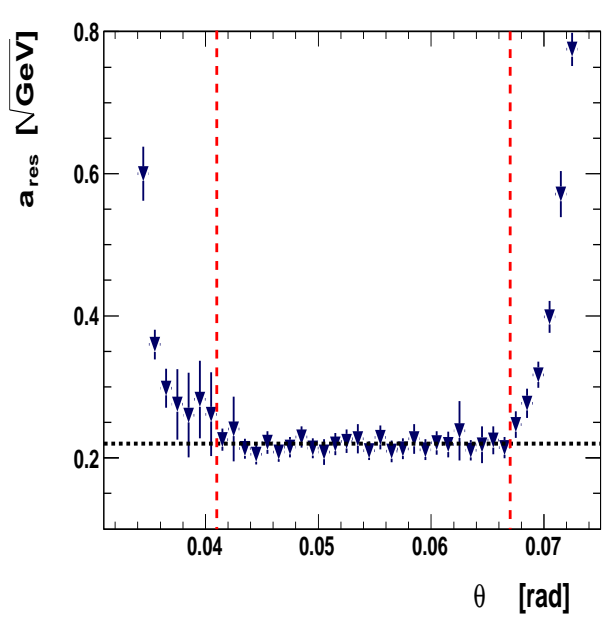

(b)

Figure 3: (a) Dependence of $(\Delta \mathrm{L} / \mathrm{L})_{\text {rec }}$, as defined in eq. (2.3), on the polar angle pad size, $1_{\theta}$. (b) The energy resolution, $\mathrm{a}_{\mathrm{res}}$, for $250 \mathrm{GeV}$ electrons as a function of the polar angle, $\theta$, covering the polar angle range of the LumiCal.

Non-zero values of $\Delta \theta$ are due to the non-linear signal sharing on finite size pads with gaps between them. The bias and the resolution in the polar angle measurement depend on the polar angle pad size. The bias causes a shift in the luminosity measurement, since events may migrate into or out of the fiducial volume. This shift reads as

$$
\left(\frac{\Delta \mathrm{L}}{\mathrm{L}}\right)_{\mathrm{rec}} \approx 2 \frac{\Delta \theta}{\theta_{\min }}
$$

Figure 3 a shows the relative shift in the luminosity as a function of the polar angular pad size, $1_{\theta}$, using the optimal value of $\mathscr{C}$. For $1_{\theta}<2 \mathrm{mrad}$ the shift in the luminosity measurement is smaller than $10^{-3}$. As the baseline for the design we have chosen $1_{\theta}=0.8 \mathrm{mrad}$, which corresponds to 64 radial divisions of the sensor. For this segmentation the polar angle resolution and bias amount to $\sigma_{\theta}=(2.2 \pm 0.01) \times 10^{-2}$ and $\Delta \theta=(3.2 \pm 0.1) \times 10^{-3} \mathrm{mrad}$, respectively. The relative shift in the luminosity is $(\Delta \mathrm{L} / \mathrm{L})_{\mathrm{rec}}=1.6 \times 10^{-4}$.

The polar angle bias needs careful understanding in test-beam measurements with sensors finally chosen for the calorimeter. Once its value is known, a correction can be applied to the luminosity measurement. The uncertainty of the luminosity measurement is then given by the uncertainty of the measured bias which may be smaller than the shift itself. The value of $1.6 \times 10^{-4}$ can therefore be considered as an upper bound on the relative luminosity bias.

With 30 radiation lengths of tungsten as absorber, high energy electrons and photons deposit almost all of their energy in the detector. The relative energy resolution, $\sigma_{\mathrm{E}} / \mathrm{E}$, is parametrised as

$$
\frac{\sigma_{\mathrm{E}}}{\mathrm{E}}=\frac{\mathrm{a}_{\text {res }}}{\sqrt{\mathrm{E}_{\text {beam }}(\mathrm{GeV})}},
$$




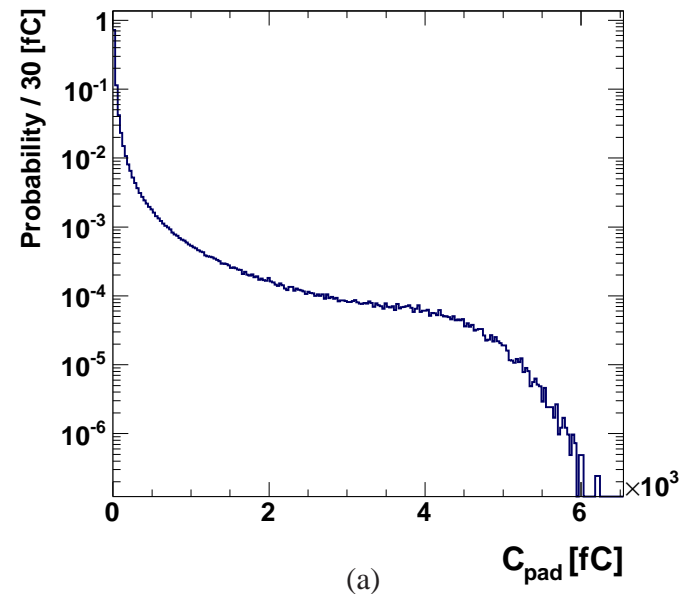

(a)

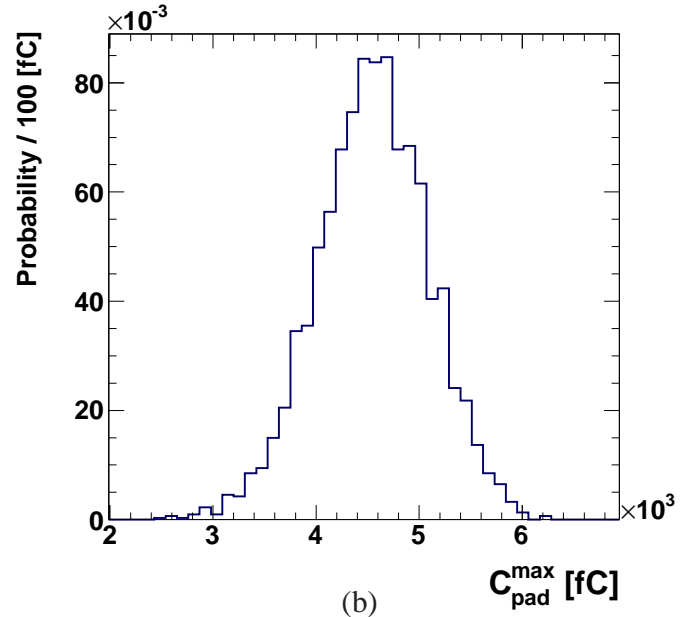

(b)

Figure 4: (a) Normalised distribution of the charge deposited in a detector pad, $\mathrm{C}_{\mathrm{pad}}$, by $250 \mathrm{GeV}$ electron showers. (b) Normalised distribution of the maximal charge collected in a single pad per shower, $\mathrm{C}_{\text {pad }}^{\max }$, for $250 \mathrm{GeV}$ electron showers.

where $\mathrm{E}$ and $\sigma_{\mathrm{E}}$ are, respectively, the central value and the standard deviation of the distribution of the energy deposited in the sensors for a beam of electrons with energy $E_{\text {beam }}$. The parameter $a_{\text {res }}$ is usually quoted as the energy resolution, a convention which will be followed here.

Figure $3 \mathrm{~b}$ shows the energy resolution as a function of the polar angle $\theta$ for electron showers with energy $250 \mathrm{GeV}$. The energy resolution parameter approaches minimal constant values between $\theta_{\min }=41 \mathrm{mrad}$ and $\theta_{\max }=67 \mathrm{mrad}$, where the shower is fully contained inside the calorimeter. The fiducial volume of LumiCal is thus defined to be the polar angular range

$$
41<\theta<67 \mathrm{mrad}
$$

as indicated by the dashed lines in figure 2a. Fiducial cuts on the minimal and maximal reconstructed polar angles of the particles used for the luminosity measurement reject events with shower leakage through the edges of LumiCal. For electron showers located inside the fiducial volume of LumiCal, the energy resolution is estimated to be $a_{\text {res }}=(0.21 \pm 0.02) \sqrt{\mathrm{GeV}}$. No dependence on the electron energy is found in the energy range from 50 to $300 \mathrm{GeV}$. In order to determine the energy of showering particles, the integrated deposited energy in the detector has to be multiplied by a calibration factor. The calibration factor is found to be constant in the same energy range.

The expected range of energy depositions in the pads has been studied for the passage of minimum ionising particles, hereafter denoted as MIPs, and for showers of $250 \mathrm{GeV}$ electrons [15]. The energy deposition in silicon is converted to released ionisation charge. The distribution of the charge in a single pad, $\mathrm{C}_{\mathrm{pad}}$, is shown in figure $4 \mathrm{a}$. It ranges between $4<\mathrm{C}_{\mathrm{pad}}<6 \times 10^{3} \mathrm{fC}$. The distribution of the maximal charge collected in a single pad is shown in figure $4 \mathrm{~b}$. About $95 \%$ of electron shower signals are less than $5.4 \times 10^{3} \mathrm{fC}$.

The impact of the digitisation of the detector signal on the LumiCal performance is investigated in ref. [15]. It is shown that an ADC with 8 bit resolution is sufficient to keep the energy resolution quoted above. No bias in the energy measurement is found. 


\subsection{BeamCal simulation studies}

BeamCal will be hit after each bunch-crossing by a large amount of beamstrahlung pairs. Their number, energy and spatial distribution depend on the beam parameters and the magnetic field inside the detector. For the nominal ILC beam-parameter set [16], beamstrahlung pairs are generated with the GUINEA-PIG program [17]. Inside the ILC detector an anti-DID field [18] is assumed. Beamstrahlung pairs are simulated in the detector, using a program based on GEANT4.

The energy deposited in the sensors of BeamCal per bunch crossing, about $150 \mathrm{GeV}$ as shown in figure 5a, and the shape of these depositions allow a bunch-by-bunch luminosity estimate and the determination of beam parameters [7]. From the spatial distribution of the deposited energy a set of observables, e.g. radial and angular moments and asymmetries, is defined. These observables are related to beam parameters like bunch sizes, emittances and bunch offsets by a matrix equation. In the single parameter determination accuracies better than $10 \%$ [7] are obtained. In the multiparameter mode correlations appear. However, reasonable precision can still be obtained by using information from other diagnostics devices.

For search experiments it is important to detect single high energy electrons on top of the wider spread beamstrahlung pairs. Superimposed on the pair depositions in figure 5a is the deposition of an electron of $250 \mathrm{GeV}$, seen as the red spot on the right side. By performing an appropriate subtraction of the pair deposits and a shower-finding algorithm which takes into account the longitudinal shower profile, high energy electrons can be detected with high efficiency, as shown in figure 5b. This feature allows to suppress the background from two-photon processes in a search e.g. for super-symmetric tau-leptons [19] in a large fraction of the parameter space.

The range of signals expected on the pads was estimated. Including the depositions from beamstrahlung signals up to $40 \mathrm{pC}$ are expected. Digitising the signals with an ADC with 10 bit resolution has no impact on the performance of the calorimeter.

GEANT4 simulations are also used to determine the expected dose and the neutron fluence in the sensors after one year of operation with nominal beam parameters. The dose in a sensor layer at the depths of the shower maximum as a function of the radius is shown in figure 6a. In the innermost ring of the calorimeter a dose of about $0.5 \mathrm{MGy}$ is expected. Since the dose is nonuniformly distributed as a function of the azimuthal angle, it approaches 1 MGy per year in some sensor areas of the inner rings.

The neutron fluence is estimated using in GEANT4 the cascade model of Bertini [20]. The fluence per year of running at nominal beam parameters is shown in figure $6 \mathrm{~b}$ as a function of the sensor layer number. Fluences up to $2 \times 10^{15}$ per layer are expected near the shower maximum. Other GEANT4 models predict lower neutron fluences, particularly at low neutron energies [21]. The distribution of the fluence of neutrons in the sensor layer with the maximum fluence is shown in figure 7. With the cascade model of Bertini, a neutron fluence of $0.4 \times 10^{12}$ neutrons per $\mathrm{mm}^{2}$ and year is expected near the beam-pipe. Albeit this is still an order of magnitude less than predicted for LHC detectors near the beam pipe dedicated tests of sensors are planned.

\subsection{Pair monitor simulations}

Additional and independent information on beam parameters will be obtained from the pair monitor [22, 23]. The device will consist of one layer of silicon pixel sensors, with pixel size of $400 \times 400$ 


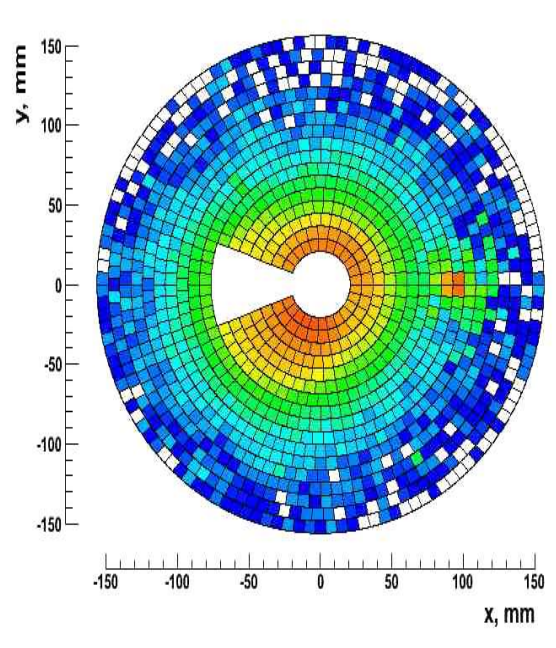

(a)

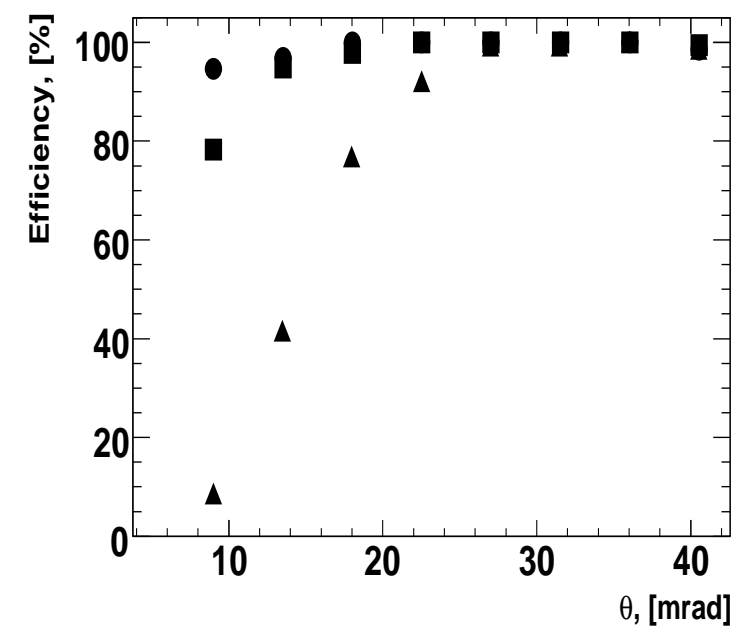

(b)

Figure 5: (a) The distribution of the energy deposited by beamstrahlung pairs after one bunch crossing in the sensors of BeamCal. The depositions are integrated over pads of $7.65 \times 7.65 \mathrm{~mm}^{2}$ area. Superimposed is the deposition of a single high energy electron (red spot on the right side). The white area in the centre allows space for the beam-pipes. (b) The efficiency to detect single high energy electrons on top of the beamstrahlung background for electron energies of 75 (triangles), 150 (squares) and 250 (circles) GeV.

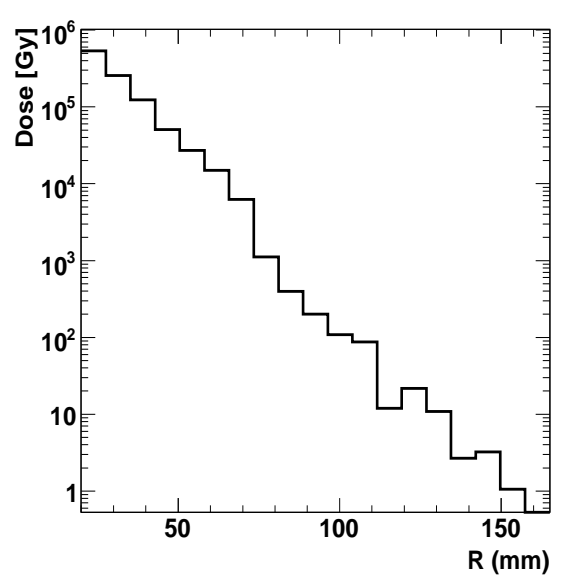

(a)

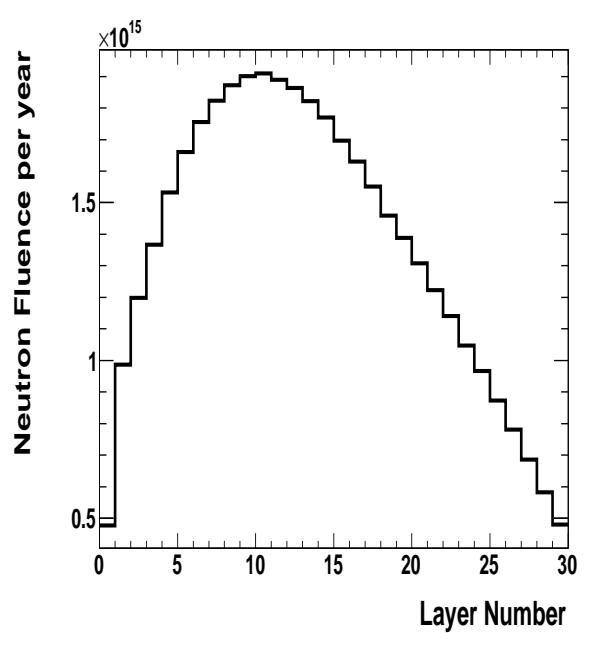

(b)

Figure 6: (a) The dose in BeamCal sensors per year as a function of the radial distance from the beam. (b) The fluence of neutrons per year inside the sensors of BeamCal as a function of the sensor layer number using the cascade model of Bertini. An integrated luminosity of $500 \mathrm{fb}^{-1}$ is assumed.

$\mu \mathrm{m}^{2}$, just in front of BeamCal to measure the number density distribution of beamstrahlung pairs. Here we investigated the sensitivity to the horizontal and vertical bunch sizes, $\sigma_{\mathrm{x}}$ and $\sigma_{\mathrm{y}}$, and the 


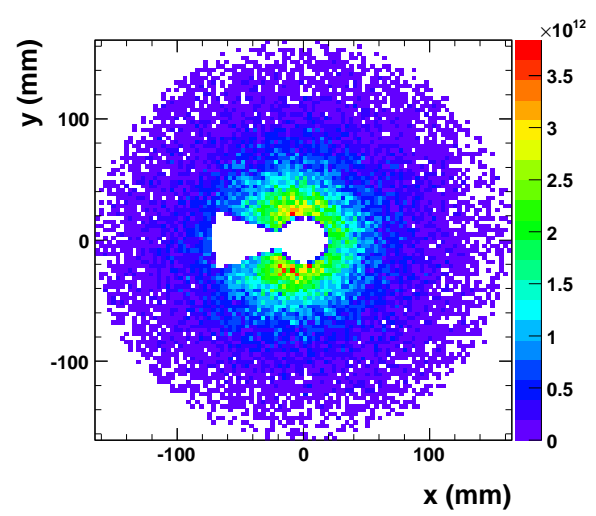

Figure 7: The fluence of neutrons per $\mathrm{mm}^{2}$ and year crossing a sensor of BeamCal near the shower maximum using the cascade model of Bertini.
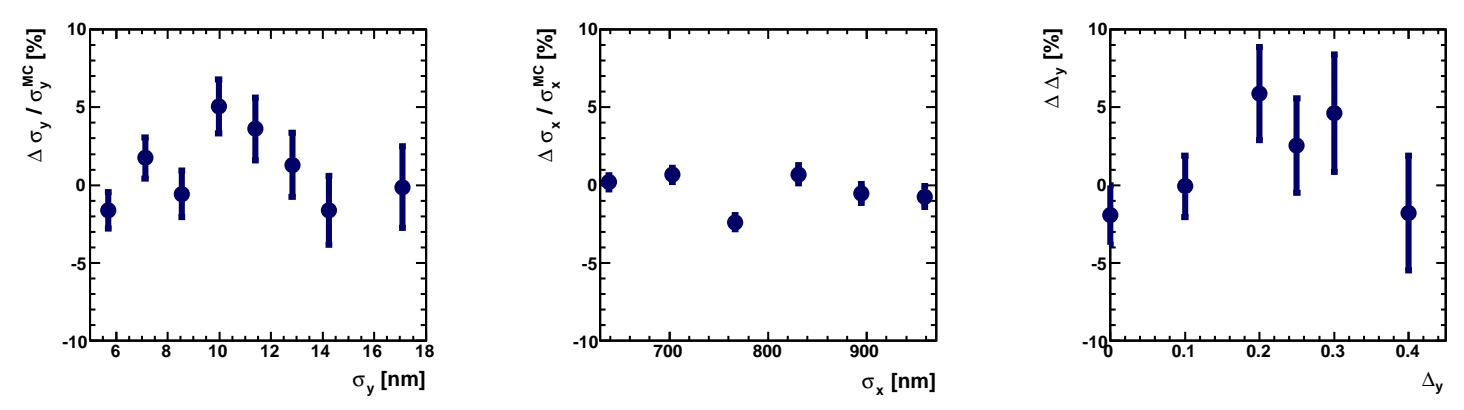

Figure 8: The relative deviations of the vertical, $\sigma_{y}$, and horizontal, $\sigma_{x}$, beam sizes, and the ratio of vertical displacement to the vertical beam size, $\Delta_{y}$, averaged over 50 bunch crossings measured by the pair monitor.

ratio of the vertical displacement between bunches crossing to their vertical size, $\Delta_{\mathrm{y}}$.

To reconstruct the beam profile several observables characterising the number density of pairs at the front face of BeamCal are used [24]. Bunch crossings are simulated for certain ranges of $\sigma_{\mathrm{x}}$, $\sigma_{\mathrm{y}}$ and $\Delta_{\mathrm{y}}$, and each of these observables is fitted with a second order polynomial. Then, several thousand bunch crossings are generated using different sets of beam parameters and $\sigma_{\mathrm{x}}, \sigma_{\mathrm{y}}$, and $\Delta_{\mathrm{y}}$ are reconstructed with the inverse matrix method. Figure 8 shows a few examples of the results displayed as the difference between the beam parameters reconstructed and set in the simulation divided by the latter, averaged over 50 bunch crossings. These quantities are compatible with zero. The relative uncertainties, averaged over about 100 such reconstructions of vertical and horizontal beam sizes and the relative vertical displacement are $10.1 \%, 3.2 \%$ and $8.0 \%$, respectively.

\section{Mechanical concepts}

On the basis of the simulation results mechanical designs of both calorimeters are developed. To allow their installation after the beam-pipe is in place, both calorimeters consist of two half-cylinders. 


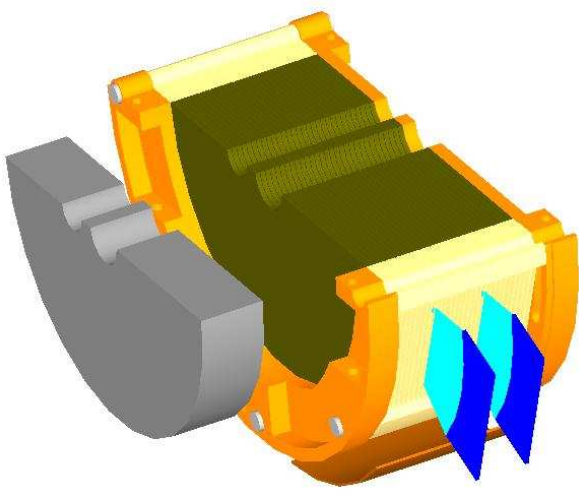

(a)

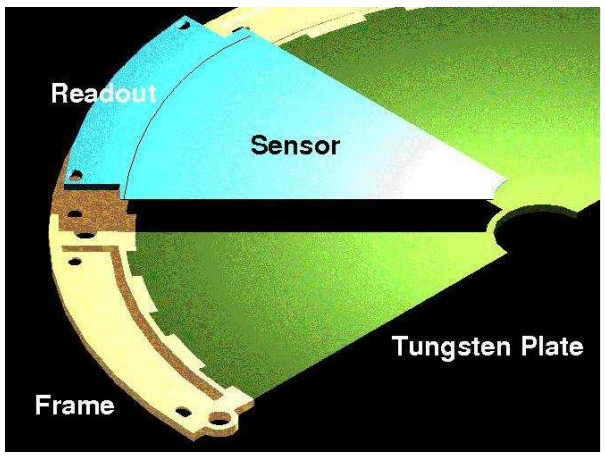

(b)

Figure 9: (a) A half-cylinder of BeamCal. The brown block is the tungsten absorber structure interspersed with sensor layers. The orange structure represents the mechanical frame. The blue segments at the outer radius indicate the front-end electronics. In front of the calorimeter a graphite shield, shown in grey, reduces the amount of low energy particles back-scattered into the tracking detectors. (b) A half-layer of an absorber disk assembled with a sensor sector and the front-end readout.

A schematic of a half cylinder of BeamCal is shown in figure 9a. The tungsten absorber disks are embedded in a mechanical frame stabilised by steel rods. Each layer is composed of a tungsten half-disc surrounded by a brass half-ring as shown in figure $9 \mathrm{~b}$. Precise holes in the brass ring will ensure a position accuracy of better than $100 \mu \mathrm{m}$. The sensors are fixed on the tungsten and connected via a flexible PCB to the front-end readout. The distance between two adjacent tungsten plates is kept to $1 \mathrm{~mm}$ to approach the smallest possible Molière radius. The sensors of BeamCal are structured into pads of about $8 \times 8 \mathrm{~mm}^{2}$ size allowing the maximum electron detection efficiency [25]. Due to the required high radiation tolerance, GaAs sensors are foreseen. For the innermost part of BeamCal, adjacent to the beam-pipes, also $\mathrm{CVD}^{1}$ diamond is considered.

The design of LumiCal is similar [26]. Since it is a precision device, special care is devoted to the mechanical stability and position control. The tungsten half-discs are held by special bolts. For a barrel structure as shown in figure 10 a finite element simulation is performed. The calorimeter weight leads to a maximal vertical displacement of $20 \mu \mathrm{m}$. For a temperature difference of $1 \mathrm{~K}$ over a disk, the deformation of the shape of the tungsten plate is estimated to be $25 \mu \mathrm{m}$. To match the requirements on the precision of the lower polar angle measurement, the sensor positions at the inner acceptance radius must be controlled to better than $40 \mu \mathrm{m}$. Other critical quantities are the distance between the two calorimeters and the position of the beam with respect to the calorimeter axis. The former must be known to about $1 \mathrm{~mm}$ and the latter to $500 \mu \mathrm{m}$. A laser based position monitoring system has been developed [27] to control the position of LumiCal over short distances with $\mu$ m precision.

For LumiCal, sensors made of high-ohmic n-type silicon are foreseen. The thickness of the sensors is about $300 \mu \mathrm{m}$. The $\mathrm{p}^{+}$side is segmented in polar and azimuthal pads and the backside

\footnotetext{
${ }^{1}$ Chemical Vapour Deposition.
} 


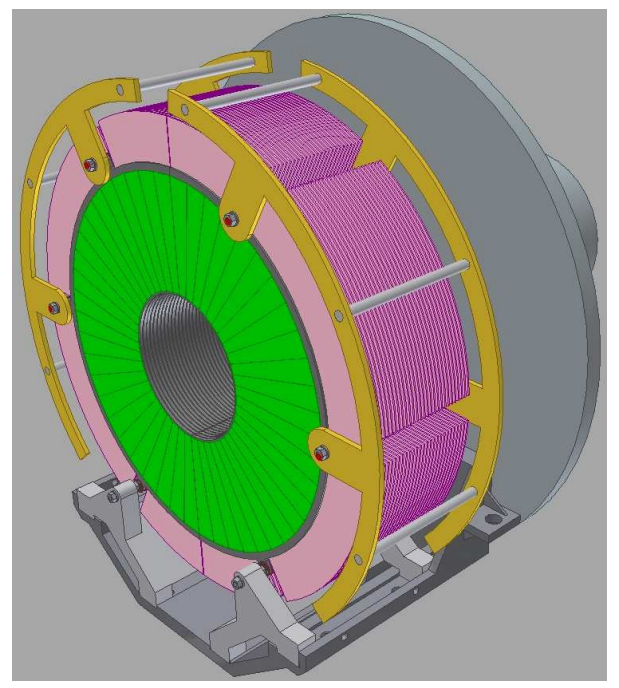

Figure 10: The mechanical structure of LumiCal. Tungsten disks are precisely positioned using 4 bolts which are stabilised by additional steel rings on both sides of the cylinder.

is fully metallised. To keep the Molière radius small the gap for the sensors is $1 \mathrm{~mm}$. The signals on the pads of both calorimeters are led by thin copper strips on a Kapton foil to the front-end electronics positioned at the outer radius of the calorimeter.

\section{Systematic effects in the luminosity measurement}

Several phenomena which may have an impact on the luminosity measurement are considered. These are: pinch effect and beamstrahlung, background from two-photon processes, the resolution and scale of the electron energy measurement and the beam polarisation.

\subsection{Pinch effect and beamstrahlung}

Due to the pinch effect the luminosity for given bunch charges and sizes will be enhanced. However, electrons and positrons may radiate photons prior to Bhabha scattering. In addition, final state particles are deflected inside the bunch. The result is a reduction of the Bhabha event counting rate in a given range of low polar angles. The reduction is found to depend on the selection criteria for Bhabha events. For a selection optimised for nominal ILC beam parameters at $500 \mathrm{GeV}$ centre-of-mass energy, it amounts to $1.51 \pm 0.05 \%$ [28], where the quoted uncertainty stems from the statistics in the simulation. The dominant contribution to the loss is due to the reduction in the centre-of-mass energy caused by beamstrahlung. The latter leads to an effective centre-of-mass energy distribution called luminosity spectrum.

In the measurement of the luminosity, the loss of Bhabha events has to be corrected for. The impact of beamstrahlung can be estimated from the measured luminosity spectrum with a relative uncertainty of about $10^{-3}$ [28]. The impact of the deflection inside the bunch depends mainly on the horizontal bunch-size, $\sigma_{\mathrm{x}}$, and the bunch length, $\sigma_{\mathrm{z}}$. Assuming that one can control these two 


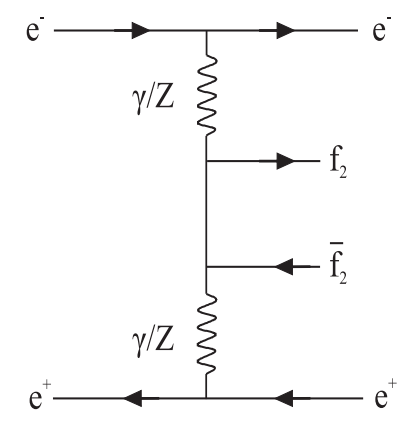

Figure 11: The Feynman graph for the dominant process in four-fermion production.

quantities with a relative uncertainty of $5 \%,{ }^{2}$ the uncertainty of a correction to the luminosity is about $1.5 \times 10^{-3}$ [28].

\subsection{Background from four-fermion production}

Four-fermion production is known to have a large cross section with maxima at low polar angles.

It is dominated by the diagram shown in figure 11, where two virtual photons are exchanged between electron spectators. We used the WHIZARD [29] event generator to obtain samples of events for final states with leptons in the inner legs. The generator was tuned to experimental data of the process $\mathrm{e}^{+} \mathrm{e}^{-} \rightarrow \mathrm{e}^{+} \mathrm{e}^{-}$c $\overline{\mathrm{c}}$ using data from LEP and other accelerators [30]. The cross-section of four-lepton production amounts to $12.0 \pm 0.5 \mathrm{nb}$ at $500 \mathrm{GeV}$ when the momenta of the exchanged photons are required to be larger than $0.1 \mathrm{GeV} / \mathrm{c}$. The spectators remain at high energy. Less than $1 \%$ of them hit the luminosity calorimeter and become a background for Bhabha events. A Bhabha event sample has been generated with a cross-section of $4.70 \pm 0.03 \mathrm{nb}$ at $500 \mathrm{GeV}$ centreof-mass energy, using the BHLUMI [31] event generator. The LumiCal response is simulated using BARBIE V4.3 [32], a GEANT3 based simulation program. The following event selection criteria are applied: the polar angle of the reconstructed shower must be within the LumiCal fiducial volume at one side and within $\theta_{\min }+4 \mathrm{mrad}$ and $\theta_{\max }-7 \mathrm{mrad}$ on the other. In addition, the total energy deposited in both calorimeters must be more than $80 \%$ of the center-of-mass energy. These criteria are optimised to reduce the impact of beamstrahlung and deflection on the Bhabha event counting to the amount given in the previous section [28]. The selection efficiency of Bhabha scattering events is about $68 \%$.

Four-fermion events in the LumiCal are to a large fraction rejected by the Bhabha selection criteria. This is illustrated in figure 12 where the hits of particles from the four-fermion final states in the front plane of LumiCal per bunch crossing are shown before and after applying the Bhabha event selection. The fraction of four-fermion final states in the selected Bhabha event sample is $2.3 \times 10^{-3}$.

At LEP energies agreement between measurements and modelling of four-fermion processes was obtained within 20\% [30]. Assuming that at $500 \mathrm{GeV}$ it will be possible to model these processes with a precision of $40 \%$, correcting the luminosity measurement correspondingly will lead to an uncertainty of $0.9 \times 10^{-3}$.

\footnotetext{
${ }^{2}$ In ref. [7] the estimated uncertainty of e.g. $\sigma_{\mathrm{x}}$ varies between $0.5 \%$ and $6.5 \%$, depending on the number of free
} 


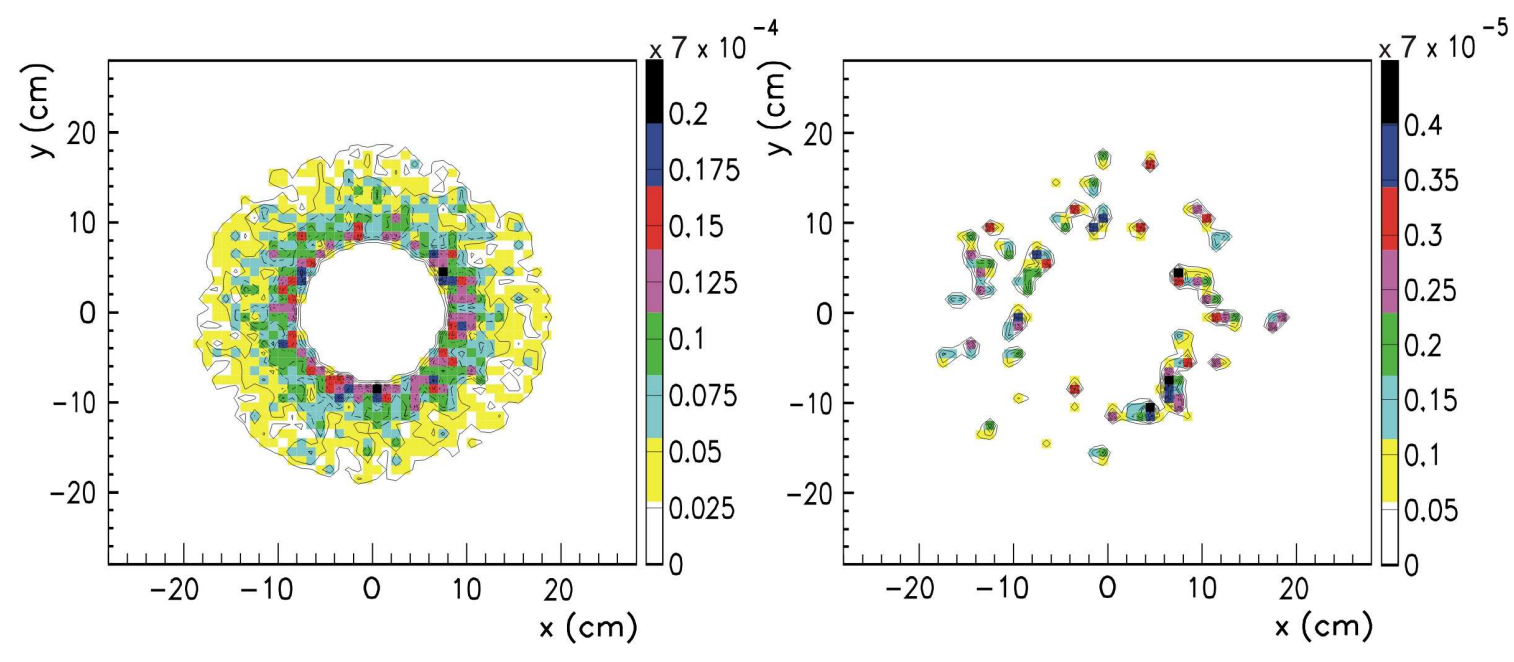

Figure 12: Average number of hits originating from four-fermion interactions per bunch crossing on the first plane of LumiCal at $500 \mathrm{GeV}$, before (left) and after (right) application of Bhabha event selection criteria.

\subsection{Effects of a bias in the energy resolution and the energy scale}

One of the criteria to select Bhabha events is the total energy measured in the calorimeters, required to be larger than $80 \%$ of the centre-of-mass energy. A possible bias in the energy resolution or the energy calibration will result in a change of the number of selected Bhabha events and hence in the measured luminosity.

The selection efficiency for Bhabha events as a function of the required energy in the calorimeters is shown in figure 13a. At the position of the cut in the measured calorimeter energy the slope of the tangent to the function is about $-1.8 \times 10^{-3}$. To keep the shift of the luminosity below $10^{-3}$, the cut in the measured calorimeter energy must be controlled with a precision of about $400 \mathrm{MeV}$. A study done allowing a constant offset in the measured energy leads to a similar requirement [33].

The effect of a bias in the energy resolution, $a_{\text {res }}$ in eq. (2.4), is illustrated in figure 13b. We estimate that if $\mathrm{a}_{\text {res }}$ can be controlled within $20 \%$, it will contribute to the luminosity uncertainty by about $10^{-4}$.

\subsection{Impact of electron and positron polarisation}

To exploit the full physics potential of a linear collider, electron and positron beams will be polarised. Polarisation will also change the Bhabha cross section in the acceptance range of LumiCal up to a few per cent [34]. In the current design the maximum values for electron and positron polarisation are 0.8 and 0.6 , respectively, with an uncertainty of 0.0025 [35]. Using these values the shift in the Bhabha cross section is $2.3 \times 10^{-2}$ with an uncertainty of $1.9 \times 10^{-4}$.

\subsection{Summary of systematic uncertainties}

In addition to effects studied in this section also the impact of the polar angle resolution and polar angle bias as estimated in section 2.1 are included. All uncertainties based on the current level

beam parameters in the analysis. A similar range of precision is obtained for $\sigma_{\mathrm{z}}$. 


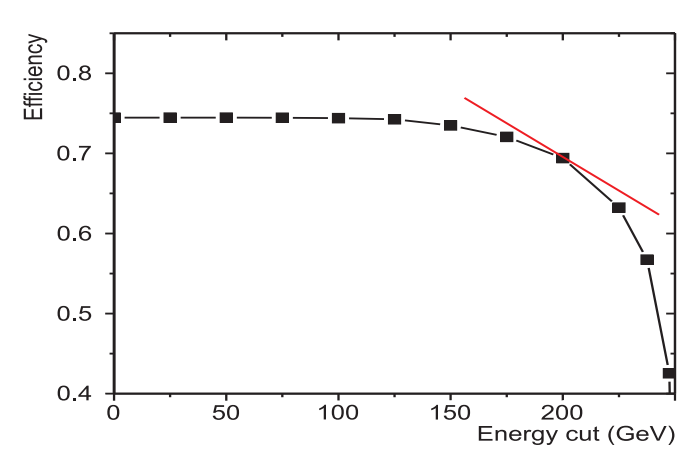

(a)

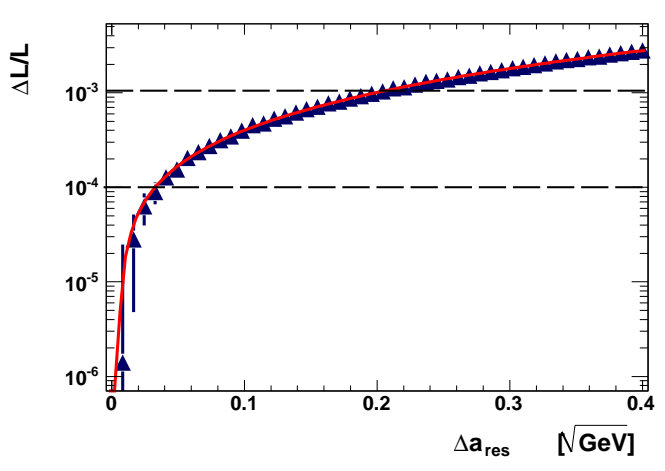

(b)

Figure 13: (a) The selection efficiency for Bhabha events as a function of the measured shower energy, (b) the shift of the measured luminosity as a function of the bias in the energy resolution parameter $\mathrm{a}_{\mathrm{res}}$.

Table 1: The estimated systematic uncertainties on the luminosity measurement from all sources considered above at a centre-of-mass energy of $500 \mathrm{GeV}$.

\begin{tabular}{|l|l|l|l|}
\hline Source & Value & Uncertainty & Luminosity Uncertainty \\
\hline$\sigma_{\theta}$ & $2.2 \times 10^{-2}$ & $100 \%$ & $1.6 \times 10^{-4}$ \\
$\Delta_{\theta}$ & $3.2 \times 10^{-3}$ & $100 \%$ & $1.6 \times 10^{-4}$ \\
$a_{\text {res }}$ & 0.21 & $15 \%$ & $10^{-4}$ \\
luminosity spectrum & & & $10^{-3}$ \\
bunch sizes $\sigma_{\mathrm{x}}, \sigma_{\mathrm{z}}$, & $655 \mathrm{~nm}, 300 \mu \mathrm{m}$ & $5 \%$ & $1.5 \times 10^{-3}$ \\
two photon events & $2.3 \times 10^{-3}$ & $40 \%$ & $0.9 \times 10^{-3}$ \\
energy scale & $400 \mathrm{MeV}$ & $100 \%$ & $10^{-3}$ \\
polarisation, $\mathrm{e}^{-}, \mathrm{e}^{+}$ & $0.8,0.6$ & 0.0025 & $1.9 \times 10^{-4}$ \\
\hline total uncertainty & & & $2.3 \times 10^{-3}$ \\
\hline
\end{tabular}

of understanding are summarised in table 1. They are considered as being uncorrelated, leading currently to a total uncertainty of $2.3 \times 10^{-3}$. The reduction of the largest uncertainty, due to the deflections of final state electrons or positrons inside the bunch, needs further investigation. Also the energy scale uncertainty may be reduced by a proper calibration.

\section{Sensor development}

\subsection{Sensors for BeamCal}

The challenge of BeamCal is to find sensors tolerating about one MGy of dose per year. So far polycrystalline CVD diamond sensors of $1 \mathrm{~cm}^{2}$ size and larger sectors of GaAs pad sensors, as shown in figure 14 , have been studied. Irradiation is done using a $10 \mathrm{MeV}$ electron beam at the S- 


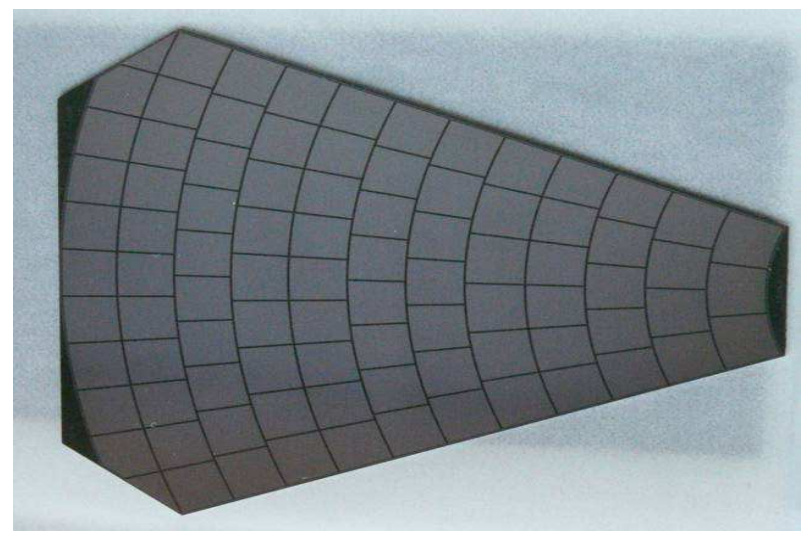

Figure 14: A prototype of a GaAs sensor sector for BeamCal with pads of about $30 \mathrm{~mm}^{2}$ area.

DALINAC accelerator [36]. The intensity is varied between 10 and $100 \mathrm{nA}$ corresponding to dose rates between 20 and $200 \mathrm{kGy} / \mathrm{h}$. Since large area CVD diamond sensors are extremely expensive, they may be used only at the innermost part of BeamCal. At larger radii GaAs sensors appear to be a promising option.

\subsubsection{GaAs sensors}

Large area GaAs sensors are obtained from the Tomsk State University. They are produced using the liquid encapsulated Czochralski method and are doped with tin and tellur as shallow donors and chromium as a deep acceptor.

Three batches with different concentrations of dopants are irradiated up to 1.2 MGy and the charge collection efficiency, CCE, is measured as a function of the absorbed dose. The results are shown in figure 15 . The charge collection efficiency depends slightly on the dopant concentration. The sensors with a lower donor concentration show a larger initial charge collection efficiency and the decrease of the charge collection efficiency as a function of the absorbed dose is less steep. The smallest decrease of the CCE as a function of the dose is observed for tin donor. A MIP signal is separated from the pedestal up to a dose of $600 \mathrm{kGy}$ for the sensors with lower donor concentration. The leakage current of a pad at room temperature before irradiation is about $200 \mathrm{nA}$ at an applied voltage of $50 \mathrm{~V}$. After exposure of a dose of $1.2 \mathrm{MGy}$ leakage currents of up to a factor 2 larger were found. The pad capacitance is measured to $12 \mathrm{pF}$. The results are consistent with previous measurements [37].

\subsubsection{CVD diamond sensors}

For polycrystalline diamond sensor samples of $1 \mathrm{~cm}^{2}$ area and $500 \mu \mathrm{m}$ thickness the linearity of the response and the leakage current and the signal collection efficiency have been investigated as a function of the absorbed dose [38]. The signal size depends linearly on the number of charged particles crossing the sensors for up to $5 \times 10^{6}$ particles in $10 \mathrm{~ns}$. The leakage current, less than $1 \mathrm{pA}$ at room temperature, depends only slightly on the absorbed dose up to $7 \mathrm{MGy}$. The charge collection efficiency rises by a factor of two for doses between 0.5 to $1 \mathrm{MGy}$, then drops smoothly 


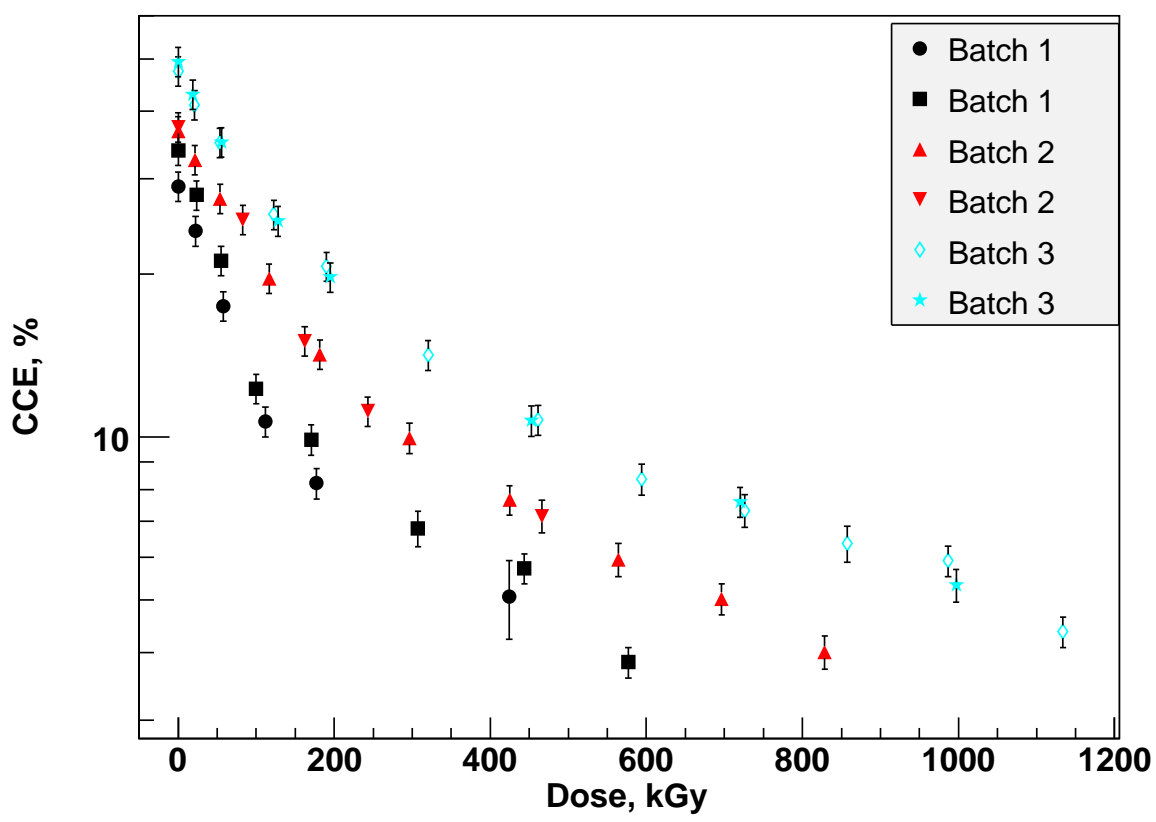

Figure 15: The CCE as a function of the absorbed dose for the GaAs sensors with different donor concentrations. The donor is tellur for batches 1 and 2 and tin for batch 3 .

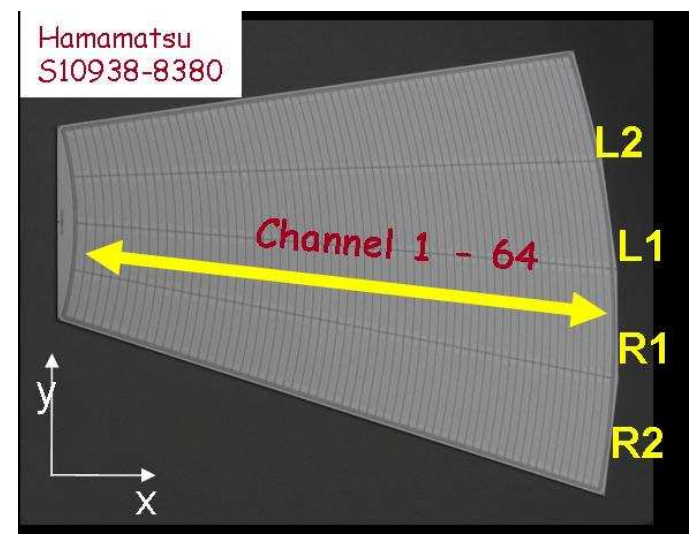

Figure 16: A prototype silicon sensor for LumiCal.

approaching the charge collection efficiency of a non-irradiated sensor. Provided the sensor is continuously irradiated, this efficiency is reached at about 7 MGy.

\subsection{Sensors for LumiCal}

Prototypes of LumiCal sensors have been designed [39] and then manufactured by Hamamatsu Photonics. A picture of a sensor is shown in figure 16. Its shape is a ring segment of $30^{\circ}$. The thickness of the n-type silicon bulk is $320 \mu \mathrm{m}$. The pitch of the concentric $\mathrm{p}^{+}$pads is $1.8 \mathrm{~mm}$ and the gap between two pads is $0.1 \mathrm{~mm}$. The leakage current of a single pad as a function of the bias voltage is shown in figure 17a. Putting the neighbouring pads on ground stabilises the measurement and reduces the current values by a factor of two. The leakage currents of all the pads of one sensor 


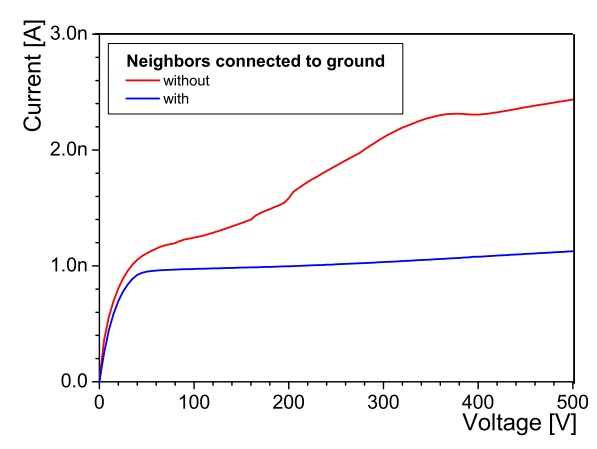

(a)

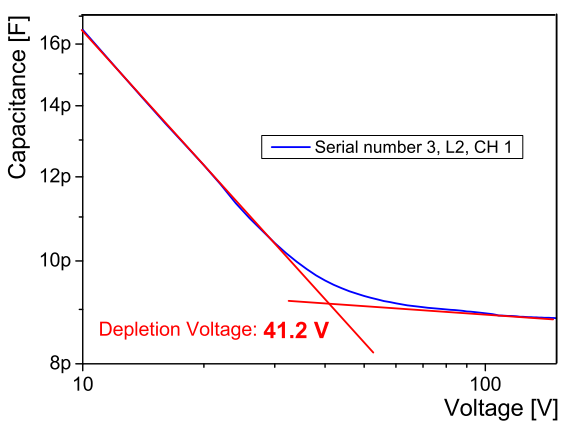

(b)

Figure 17: (a) The dependence of the leakage current on the bias voltage for a single pad with and without grounded neighbours. (b) The capacitance of a pad as a function of the bias voltage.

have been measured at a bias voltage of $500 \mathrm{~V}$. All pads except one have a leakage current in the range from 1 to $4 \mathrm{nA}$. Less than 5\% of all pads have a break-through voltage below $500 \mathrm{~V}$. For other sensors the results are similar. The capacitance as a function of the bias voltage for a pad is shown in figure 17b. Also shown is how the value of the full depletion voltage is obtained. Values from $39 \mathrm{~V}$ to $43 \mathrm{~V}$ were found. At a voltage of $100 \mathrm{~V}$ the pad capacitance values are between $8 \mathrm{pF}$ for the smallest pads and $25 \mathrm{pF}$ for the largest pads.

\section{ASIC developments}

Since the occupancy in BeamCal and LumiCal is relatively large they must be read-out after each bunch crossing. Therefore special front-end and ADC ASICs have been developed which match the timing of the ILC-bunch trains with a frequency of $5 \mathrm{~Hz}$ and about one ms duration with 300 ns between bunches. Since the ASICs are positioned at the outer radius of the calorimeters the expected radiation dose is noncritical. From Monte Carlo simulations less than $140 \mathrm{~Gy}$ and about one Gy are estimated for BeamCal and LumiCal, respectively, for one year of operation at $500 \mathrm{GeV}$ centre-of-mass energy and nominal beam parameters.

\subsection{LumiCal readout}

The design of the LumiCal front-end electronics was performed for the proposed detector architecture [40]. The front-end ASIC is supposed to work in two modes, the physics mode and the calibration mode. In the physics mode, electromagnetic showers will be measured with large energy depositions on the pads. The front-end ASIC must process signals up to at least $6 \mathrm{pC}$ per channel. In the calibration mode, MIP signals from single relativistic muons will be measured. The minimum size of these signals is $2 \mathrm{fC}$, corresponding to the low end of the Landau distribution for MIPs in $300 \mu \mathrm{m}$ thick silicon. From the sensor segmentation a range of pad capacitances between $10 \mathrm{pF}$ and $100 \mathrm{pF}$ was obtained. ${ }^{3}$ Because of the high expected occupancy, the frontend ASIC needs to be fast enough to resolve signals from subsequent bunch crossings which are separated in time by about $300 \mathrm{~ns}$.

\footnotetext{
${ }^{3}$ The sensor segmentation was revised later, resulting in pad capacitances between $10 \mathrm{pF}$ and $25 \mathrm{pF}$.
} 


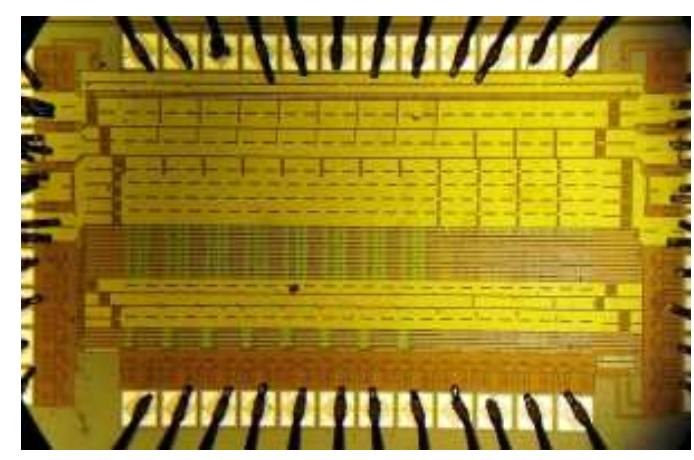

(a)

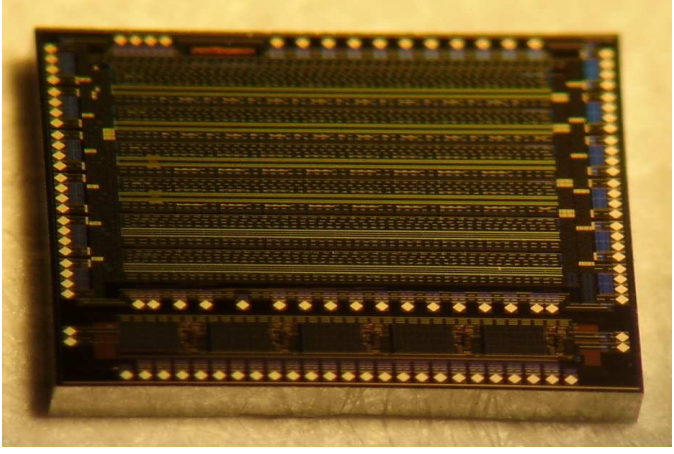

(b)

Figure 18: Photograph of prototypes of the front-end ASIC (a) and the ADC ASIC (b)

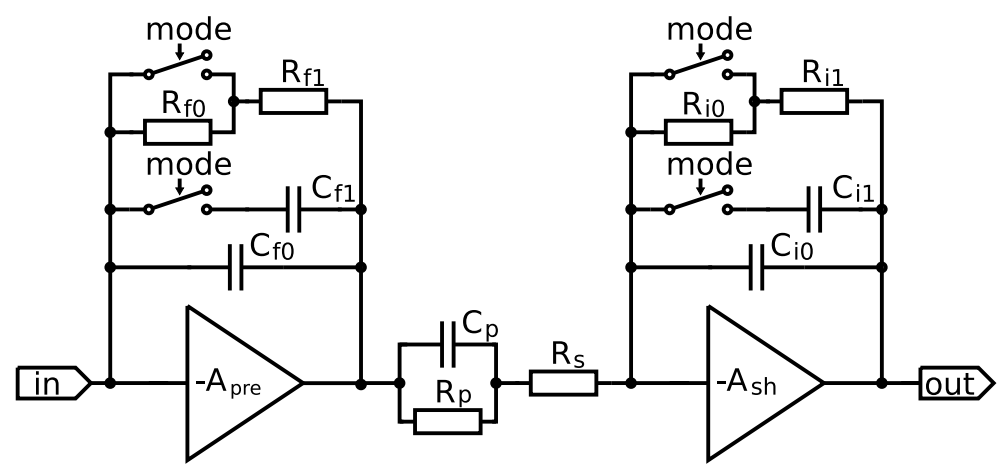

Figure 19: Block diagram of the single front-end channel

The simulations of LumiCal indicate that the shower reconstruction needs at least 8 bit precision. Severe requirements set on the readout electronics power dissipation may be strongly relaxed if switching of the power between bunch trains is done. This is feasible since in the ILC experiments after each $1 \mathrm{~ms}$ bunch train there will be a pause of about $200 \mathrm{~ms}$ [16].

The prototype ASICs, as shown in figures $18 \mathrm{a}$ and $18 \mathrm{~b}$, are fabricated in $0.35 \mu \mathrm{m}$ CMOS technology.

\subsubsection{Front-end electronics design}

The chosen front-end architecture comprises a charge sensitive amplifier, a pole-zero cancellation circuit (PZC) and a shaper, as shown in figure 19. In order to cope with large charges in the physics mode and small ones in the calibration mode a variable gain in both the charge amplifier and the shaper is applied. The mode switch in figure 19 changes the effective values of the feedback circuit components $R_{f}, C_{f}, R_{i}, C_{i}$ and therefore the transimpedance gain of the front-end ASIC is changed. The low gain (large $\mathrm{C}_{\mathrm{f}}$ ) is used for the physics mode when the front-end processes signals from

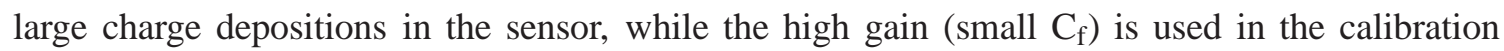
mode. Assuming high enough open loop gain of the pre-amplifier $\left(\mathrm{A}_{\text {pre }}\right)$ and the shaper amplifier 


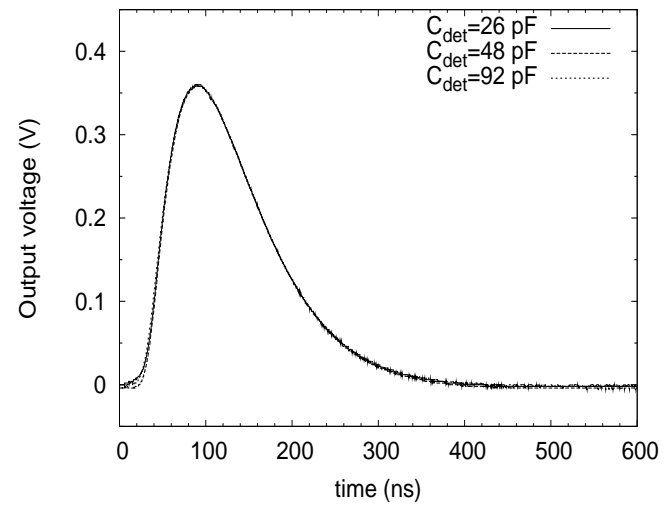

(a)

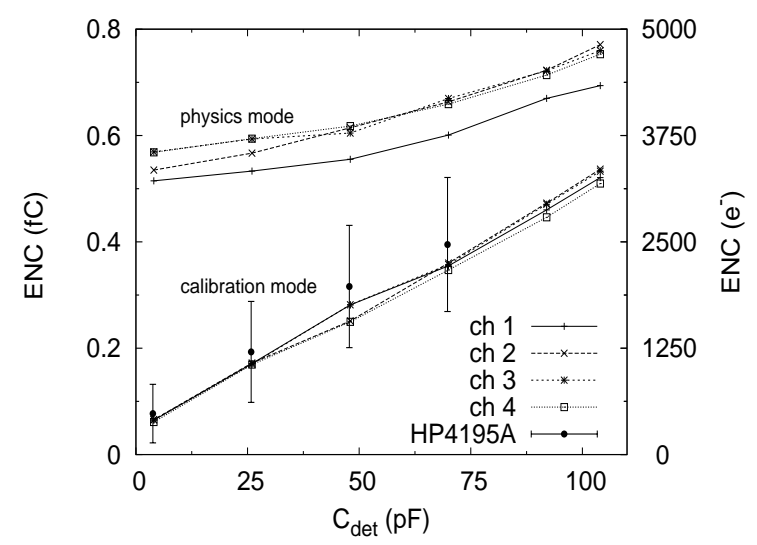

(b)

Figure 20: (a) Output pulses in physics mode as a function of the input capacitance for $\mathrm{Q}_{\text {in }}=3.3 \mathrm{pC}$. (b) Noise ENC measurements obtained with true r.m.s. meter for the front-end ASIC.

$\left(\mathrm{A}_{\mathrm{sh}}\right)$, the transfer function of this circuit is given by

$$
\frac{\mathrm{U}_{\text {out }}(s)}{\mathrm{I}_{\text {in }}(s)}=\frac{1}{\mathrm{C}_{\mathrm{f}} \mathrm{C}_{\mathrm{i}} \mathrm{R}_{\mathrm{s}}} \cdot \frac{s+1 / \mathrm{C}_{\mathrm{p}} \mathrm{R}_{\mathrm{p}}}{s+1 / \mathrm{C}_{\mathrm{f}} \mathrm{R}_{\mathrm{f}}} \cdot \frac{1}{\left(s+1 / \mathrm{C}_{\mathrm{i}} \mathrm{R}_{\mathrm{i}}\right)\left(s+1 / \mathrm{C}_{\mathrm{p}}\left(\mathrm{R}_{\mathrm{p}}|| \mathrm{R}_{\mathrm{s}}\right)\right)} .
$$

By setting properly the PZC parameters $\left(\mathrm{C}_{\mathrm{f}} \mathrm{R}_{\mathrm{f}}=\mathrm{C}_{\mathrm{p}} \mathrm{R}_{\mathrm{p}}\right)$ and by equalising the shaping time constants $\left(C_{i} R_{i}=C_{p}\left(R_{p} \| R_{s}\right)\right)$, one obtains the first order shaping, equivalent to a CR-RC filter, with a peaking time $\mathrm{T}_{\text {peak }}=\mathrm{C}_{\mathrm{i}} \mathrm{R}_{\mathrm{i}}$. A simple first order shaping is chosen as a trade-off between the noise and the power dissipation. Regarding the noise, the main requirement is to obtain in calibration mode the signal to noise ratio of about 10 for the largest sensor capacitances. Both of the amplifying stages $\left(\mathrm{A}_{\mathrm{pre}}, \mathrm{A}_{\mathrm{sh}}\right)$ are designed as folded cascodes [41] with active loads, followed by source followers. In the prototype ASIC, eight front-end channels are implemented. A more detailed discussions of the front-end ASICs can be found in ref. [42].

\subsubsection{Front-end electronics measurements}

Figure 20a shows the response of the front-end channel to charge injected through the input test capacitance for different values of the input capacitance, $\mathrm{C}_{\mathrm{det}}$, within the interesting range. The sensor capacitance is simulated with an external capacitor. It is seen that both the amplitude and the peaking time are not sensitive to the value of the input capacitance in agreement with HSPICE simulations.

The output noise has been measured using a HP3400 true r.m.s. meter [43]. The equivalent noise charge, ENC, as a function of input capacitance is shown in figure 20b. Results obtained for the physics and calibration modes are shown on the same plot. Since the HP3400 bandwidth is only up to $10 \mathrm{MHz}$ the numbers may be underestimated by about 20\%. The measured ENC as a function of $\mathrm{C}_{\mathrm{det}}$ are in agreement with simulations. In particular, in the calibration mode the signal to noise ratio of 10 is maintained for input capacitances up to about $100 \mathrm{pF}$. For a few points additional noise measurements have been performed by measuring the output noise spectra using a HP4195A 


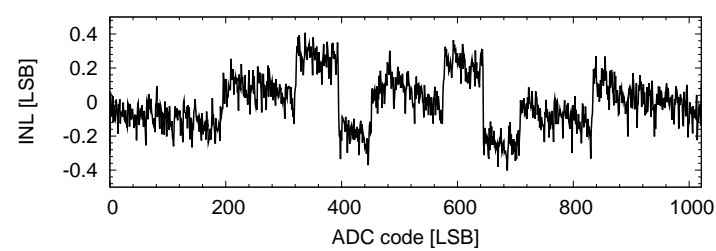

(a)

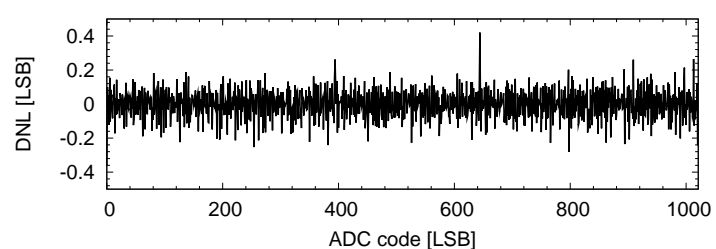

(b)

Figure 21: Static measurements of (a) INL and (b) DNL at $20 \mathrm{MHz}$ sampling frequency.

spectrum analyser [43] and then integrating it numerically. The results of such measurements are added in figure 20b. They agree within their uncertainties with the HP3400 measurements.

In order to test the effectiveness of the PZC circuit, the front-end response has been measured as a function of the rate of input pulses. To avoid input charges of both polarities when using a square-wave test signal, the staircase test waveforms are synthesised using the Tektronix AWG2021 waveform generator. It was found that the change in amplitude reaches $2 \%$ for input rates of about $3 \mathrm{MHz}$ and is quite insensitive to the input capacitance. The power consumption of about $8.9 \mathrm{~mW} /$ channel is measured in accordance with expectations from simulation.

\subsubsection{ADC design}

As a compromise between speed, area and power consumption the ADC was designed using pipeline technology. A 1.5-bit per stage architecture is chosen because of its simplicity and immunity to the offsets in the comparator and amplifier circuits. The prototype ADC consists of an input sample and hold circuit, 9 pipeline stages and digital correction circuitry. In addition, the power switching feature is also implemented. More details about the ADC design can be found in ref. [44].

\subsubsection{ADC performance measurements}

The static measurements of the Integral Nonlinearity, INL, and the Differential Nonlinearity, DNL, obtained at a sampling frequency of $20 \mathrm{MHz}$, are shown in figures $21 \mathrm{a}$ and $21 \mathrm{~b}$, respectively. These parameters are calculated using the histogramming method. The measured INL is always less than $1 \mathrm{LSB}$ while the DNL is below 0.5 LSB. These results attest to a very good ADC linearity. To estimate the dynamic performance, measurements with sinusoidal wave input are performed [45]. An example of a measured Fourier spectrum using a $1.8 \mathrm{MHz}$ full scale $(0 \mathrm{~dB})$ input signal sampled at $20 \mathrm{MHz}$ is shown in figure 22a. It is seen that the noise and harmonic components are small enough not to affect significantly the resolution. The signal to noise ratio, SNHR, is measured as a function of sampling frequency as shown in figure 22b. An SNHR of about $58 \mathrm{~dB}$ is obtained in the frequency range up to almost $25 \mathrm{MHz}$.

\subsection{BeamCal readout}

The BeamCal ASIC, designed for $180 \mathrm{~nm}$ TSMC technology, will be able to handle 32 channels. The two modes of operation require a front-end circuit capable of a wide performance envelope: high slew rate for standard data taking, and low noise for calibration. In standard data taking the occupancy is high, and therefore all data from a full bunch train must be recorded, to be read out between bunch trains. Because of its reliability, density and redundancy, a digital memory array will 


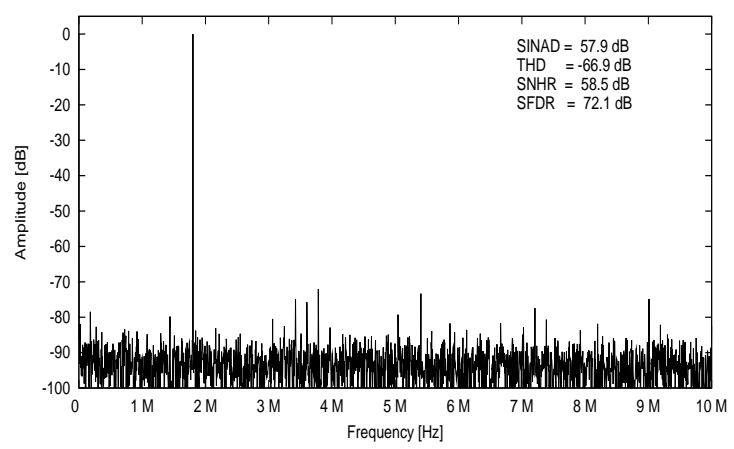

(a)

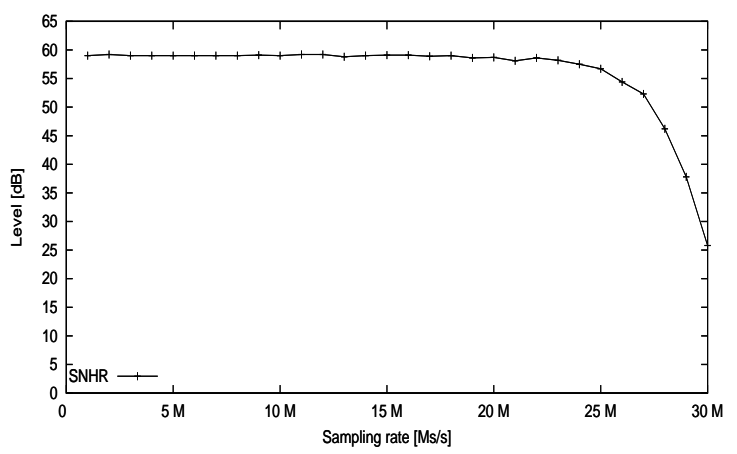

(b)

Figure 22: (a) Example of the Fourier spectrum measurement with $\mathrm{f}_{\text {in }}=1.8 \mathrm{MHz}$ and $\mathrm{f}_{\mathrm{clk}}=20 \mathrm{MHz}$, (b) SNHR as a function of the sampling rate.

be used to store the data from all collisions in each bunch train. This choice requires a sampling rate of 3.25 MHz per channel, which is achieved by 10-bits, successive approximation analog-to-digital converters [46]. The small size of this ADC architecture allows to use one converter per channel.

In this front-end ASIC, the dominant noise source is the charge sensitive amplifier series noise. Assuming $40 \mathrm{pF}$ input capacitance, high occupancy and the $300 \mathrm{~ns}$ period, a careful design of noise filtering and baseline restoration is necessary [47].

In order to take advantage of all the time available for signal processing, the filter for calibration operation has been implemented using switched-capacitor, SC, circuits [48]. This technique allows to precisely define the circuit time constants depending on the input clock frequency and the ratio of two capacitors. Baseline restoration is achieved by means of a fast gated reset, followed by a slow reset-release technique to reduce the effect of a split doublet. The slow reset-release is implemented using SC circuits.

In standard data taking operation, an adequate noise power is effectively achieved by means of a slow reset-release technique, similar to that used in calibration operation. An explicit filter for standard data taking operation is unnecessary, as the amplifier bandwidth suffices for noise filtering purposes.

Figure 23 shows a simplified block diagram for a single channel. In standard data taking operation, since filtering is unnecessary, the integrator is bypassed to reduce power consumption.

For design purposes, the transistor-level noise analysis has been carried out using the $g_{m} / I_{D}$ technique [49], which takes noise coefficients directly from SPICE simulation results. As this is a gated front-end, the system-level noise analysis has been done using the weighting function approach.

Since the system's dominant noise source is series noise, a triangular-shaped weighting function effectively minimises the output noise power. The negative slope section of the triangular weighting function is easily implemented by means of an integrator - in this case, a SC integrator. The positive slope section is achieved by means of the slow reset-release technique mentioned earlier. The weighting function resulting from an ideal reset-release and a SC integrator is shown in figure 24, left; a more realistic weighting function, reconstructed from SPICE simulation results, is shown in the right plot. In both cases, the target noise level is effectively achieved. 


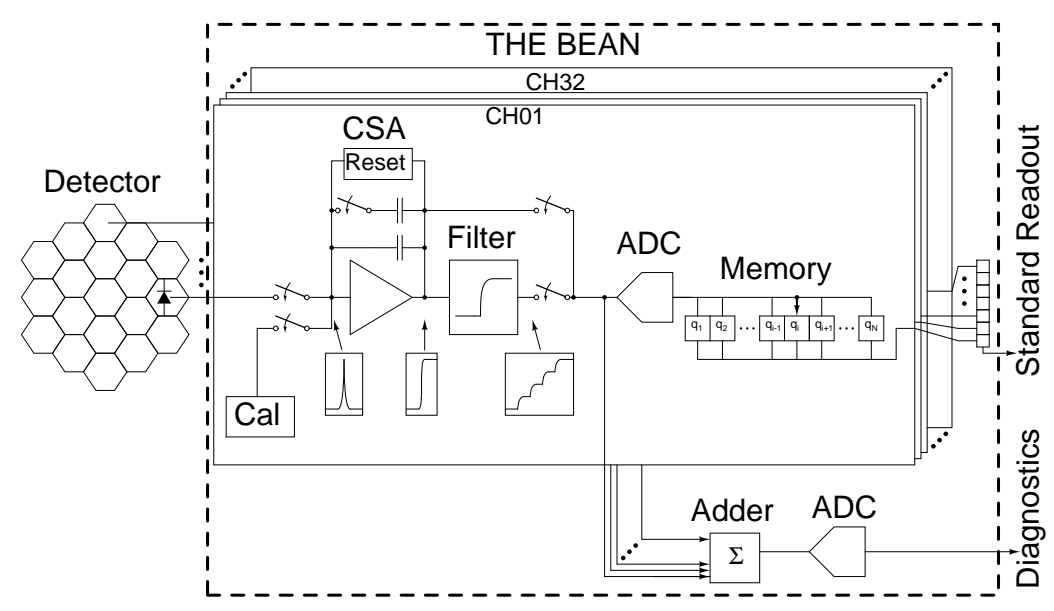

Figure 23: Simplified BeamCal ASIC block diagram of a single channel. In addition to the standard read-out a fast analog sum of groups of pads for beam-tuning is delivered by the Adder.
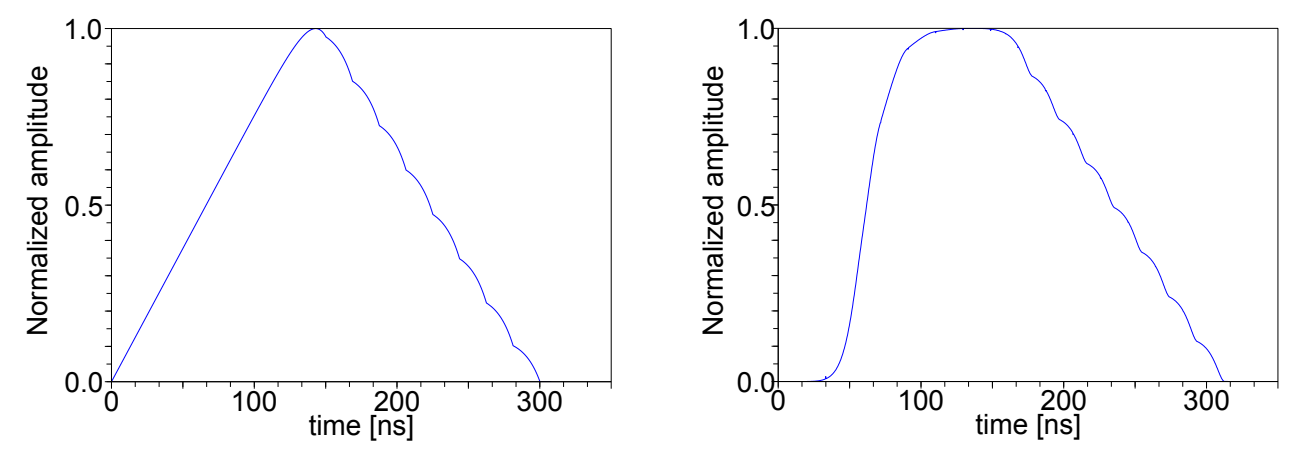

Figure 24: Front-end weighting function assuming ideal components (left) and simulation results (right) in the calibration mode

\subsubsection{Circuit implementation}

The charge sensitive amplifier is a folded-cascode amplifier with NMOS input device, connected to a switched-capacitor feedback network. The amplifier input transistor is biased at $450 \mu \mathrm{A}$ whereas the load works at about $50 \mu \mathrm{A}$. The feedback network consist of two feedback capacitors of 0.9 $\mathrm{pF}$ and $44.1 \mathrm{pF}$ for calibration and standard data taking modes, respectively. Both have a reset transistor, with a gate voltage driven by the switched-capacitor reset-release network. The amplifier output is pseudo-differential.

In order to isolate the amplifier from the filter's SC-related kickback noise, a buffer circuit is used. The buffer also allows signal shifting, producing a more adequate common-mode level for the filter. The buffer consumes $130 \mu \mathrm{A}$ and consists of a source follower, with cascoded current source and an additional device to keep a nearly constant operational point in the input transistor. This serves the purpose of enhancing the buffer linearity.

The filter implemented is a fully-differential switched-capacitor integrator. Capacitor values were carefully designed in order to obtain the adequate noise performance. The core of the inte- 


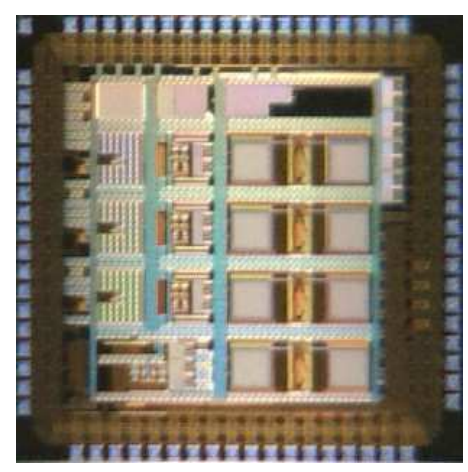

Figure 25: BeamCal Instrumentation ASIC Prototype

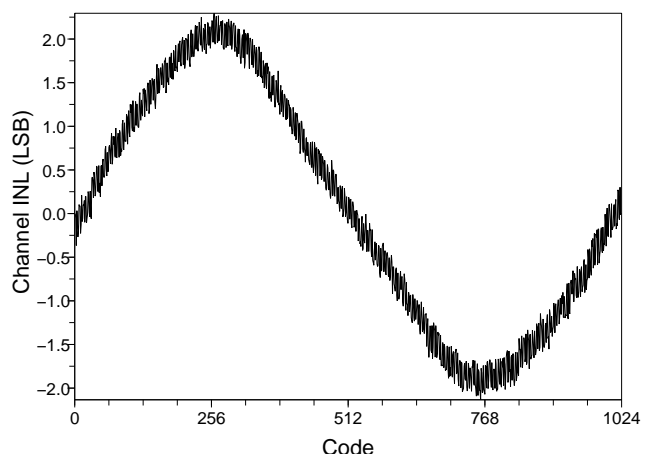

(a)

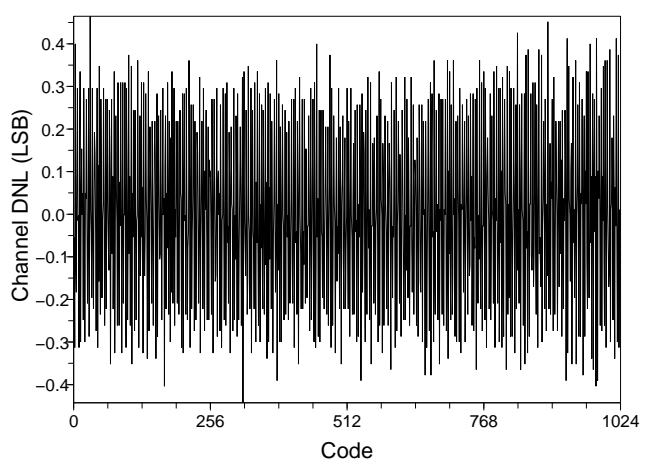

(b)

Figure 26: Results of (a) the INL and (b) the DNL using $2 \mathrm{fF}$ unit capacitors.

grator is a class $\mathrm{A} / \mathrm{AB}$ amplifier [50] that consumes $456 \mu \mathrm{A}$.

The converter is a 10-bit, fully-differential successive approximation register ADC. The one included in the BeamCal ASIC has $16 \mathrm{fF}$ unit capacitances, and similar versions with $4 \mathrm{fF}$ and $2 \mathrm{fF}$ unit capacitances were also designed for individual characterisation.

The BeamCal ASIC prototype, similar to the ASIC described in figure 23, but including only three channels and no internal memory, was fabricated and is currently being tested. Figure 25 shows the $2.4 \mathrm{~mm} \times 2.4 \mathrm{~mm}$ die.

\subsubsection{Test results}

The ADC in the BeamCal ASIC has been quantitatively characterised, along with the additional versions of the $\mathrm{ADC}$ using smaller unit capacitances. Figures $26 \mathrm{a}$ and $26 \mathrm{~b}$ show the INL and DNL for the ADC using $2 \mathrm{fF}$ capacitors. The measurements were done at the nominal sampling frequency of $3.125 \mathrm{MHz}$. The ADC input was a ramp, generated by 16-bit DAC, and the static performance measurements were calculated using the histogram method on the ADC digital output. The results are consistent with unit capacitance matching better than $0.1 \%$. The INL cubic-like shape in figure 26a is explained due to copper dishing effects, and will be corrected in future versions by re-arranging the capacitor array connections. 


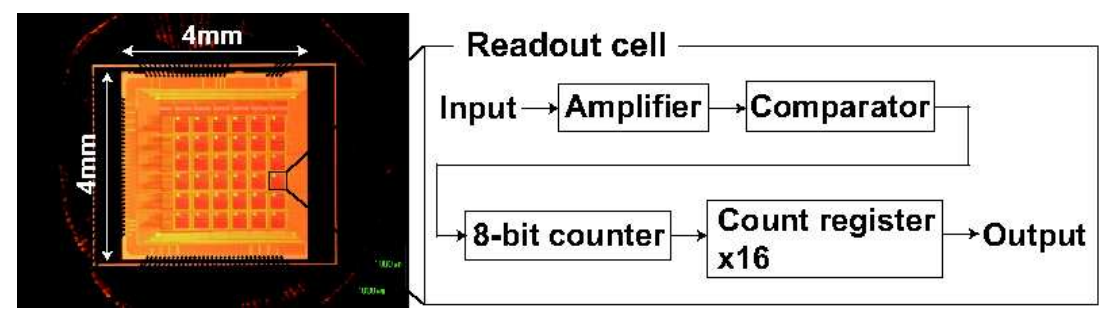

Figure 27: Picture of the prototype of the pair monitor readout ASIC and schematic diagram of the circuit in a readout cell. The readout cell consists of the amplifier, comparator, 8-bit counter, and 16 count-registers.

\subsection{Pair monitor readout}

A prototype ASIC has been designed with 36 readout cells arranged as an array of $6 \times 6$, as shown in figure 27. Each cell has an amplifier block, comparator, an 8-bit counter and a 16 count-registers. The amplifier block consists of a charge sensitive pre-amplifier, a threshold block and a differentialamplifier. The pre-amplifier is a constant-current feedback-type amplifier. The time-over-threshold of the output signal is proportional to the injected charge through the constant current feedback in the pre-amplifier. In the 8-bit counter, the Gray code is used to count the number of hits. The 16 count-registers are prepared to store hit counts in one bunch train subdivided in 16 time slices. There are also decoders which select a count-register to store and readout the hit count. A shift register to select a readout pixel, data transfer to the output line and distributor of the operation signals are arranged around the 36 readout cells as a glue logic. The bonding pad is prepared in each cell to be attached to a sensor with bump bonding. The prototype ASIC has been produced with TSMC $250 \mathrm{~nm}$ CMOS process. The chip size is $4 \times 4 \mathrm{~mm}^{2}$, and the readout cell size is $400 \times 400 \mu \mathrm{m}^{2}$.

Figure 28 shows the response of the counter block. The state of the counter bits changes at each test pulse indicating a bunch crossing. The number of hits is measured in 16 time slices of a bunch train. The data stored will then be read-out during the inter-train time. The test is performed counting the hits in each time slice with a count rate of $4 \mathrm{MHz}$, larger than expected at the ILC. The number of hits was counted without any bit lost.

We also studied the noise level in the circuit. The count efficiency was investigated as a function of the threshold voltage at the comparator. Fitting the efficiency curve with the error function, a standard deviation of $0.94 \mathrm{mV}$ was obtained. With the gain of $1.6 \times 10^{-3} \mathrm{mV}$ per electron, this corresponds to an ENC of about 600 electrons.

As the next step, a pair-monitor prototype will be built in Silicon On Insulator technology. The sensor and readout ASIC will be prepared on the same wafer. This prototype will be used to investigate not only the standard characteristics but also the radiation tolerance. Currently, an ASIC is developed in OKI $0.2 \mu \mathrm{m}$ FD-SOI CMOS [51] technology. 


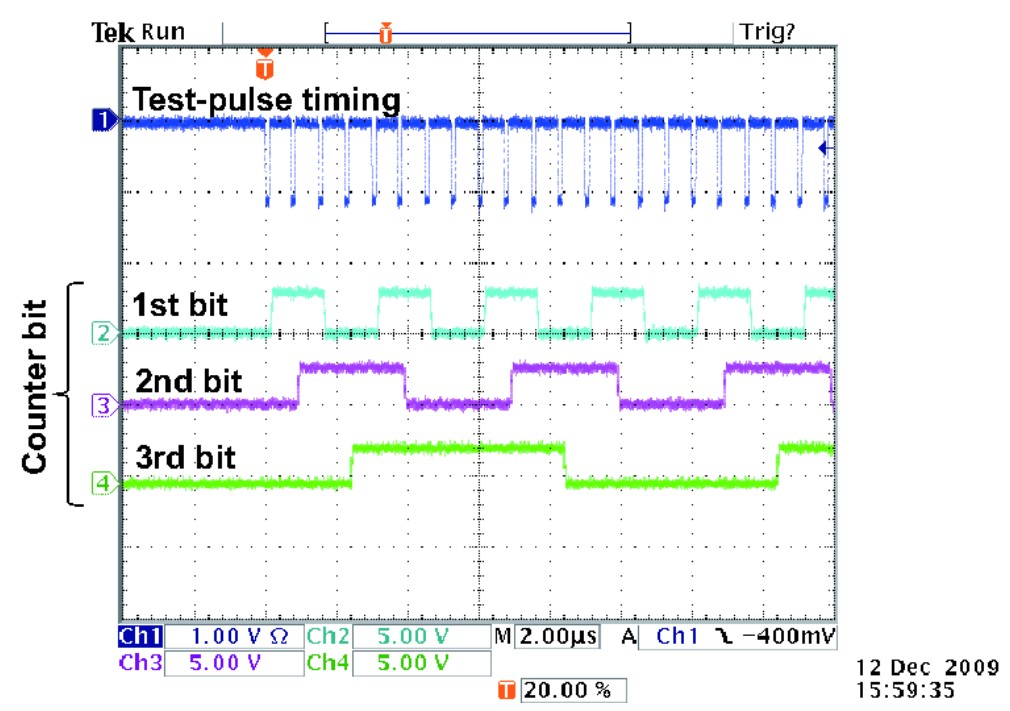

Figure 28: Output signals from the counter block. The lower 3 bits of the 8-bit counter are shown. The test-pulse timing corresponds to the bunch crossing frequency if the ILC.

\section{Summary}

A design for the instrumentation of the very forward region of a detector at the International Linear collider is presented. Two calorimeter are planned, LumiCal to measure precisely the luminosity and BeamCal, supplemented by a pair monitor, for a fast luminosity estimate and beam tuning. Both calorimeters extend the coverage of the detector to small polar angles. Parameters relevant for the physics program have been estimated by Monte Carlo simulations and found to match the requirements for the chosen geometry. Prototypes of the major components such as sensors, frontend ASICs and ADC ASICs are developed, produced and tested. Their measured performance fulfils the specifications derived from the Monte Carlo simulations. The results presented here demonstrate that the sensors and the ASICs are ready to be integrated into a fully functional prototype detector and to perform, as the next step, tests of fully assembled sensor plane prototypes.

\section{Acknowledgments}

This work is supported by the Commission of the European Communities under the $6^{\text {th }}$ Framework Program "Structuring the European Research Area", contract number RII3-026126. Tsukuba University is supported in part by the Creative Scientific Research Grant No. 18 GS0202 of the Japan Society for Promotion of Science. The AGH-UST is supported by the Polish Ministry of Science and Higher Education under contract Nr. 372/6.PRUE/2007/7. The INP PAN is supported by the Polish Ministry of Science and Higher Education under contract Nr. 141/6.PR UE/2007/7. IFIN-HH is supported by the Romanian Ministry of Education, Research and Innovation through the Authority CNCSIS under contract IDEI-253/2007. The VINCA group is benefiting from the project "Physics and Detector R\&D in HEP Experiments" supported by the Ministry of Science of 
the Republic of Serbia. J. Aguilar, P. Ambalathankandy and O. Novgorodova are supported by the 7th Framework Programme "Marie Curie ITN", grant agreement number 214560.

\section{References}

[1] International Linearcollider Reference Report (2007), http://www.linearcollider.org/about/Publications/Reference-Design-Report.

[2] The Compact Linear Collider Study, http://clic-study.web.cern.ch/clic-study/.

[3] T. Abe et al., The International Large Detector: Letter of Intent, FERMILAB-LOI-2010-01, FERMILAB-PUB-09-682-E, DESY-2009-87, KEK-REPORT-2009-6, arXiv:1006.3396 (2010).

[4] E.L. Berger et al., SiD Letter of Intent (2009), https://confluence.slac.stanford.edu/display/SiD/home.

[5] H. Abramowicz et al., Instrumentation of the very forward region of a linear collider detector, IEEE Trans.Nucl.Sci. 51 (2004) 2983.

[6] K. Mönig, Physics needs for the forward region, in V. Workshop: Instrumentation of the Forward Region of a Linear Collider Detector, August, 26-28 (2004) DESY, Zeuthen, Germany, http://www-zeuthen.desy.de/lcdet/Aug_04_WS/aug_04_ws.html.

[7] Ch. Grah and A. Sapronov, Beam parameter determination using beamstrahlung photons and incoherent pairs, 2008 JINST 3 P10004.

[8] R. Bonciani and A. Ferroglia, Bhabha Scattering at NNLO,

Nucl. Phys. B Proc. Suppl. 181-182 (2008) 259;

T. Becher and K. Melnikov, JHEP 06 (2007) 084;

S. Actis, M. Czakon, J. Gluza and T. Riemann, Fermionic NNLO contributions to Bhabha scattering, Acta Phys.Polon. B 38 (2007) 3517;

A.A. Penin, Two-loop photonic corrections to massive Bhabha scattering, Nucl. Phys. B 734 (2006) 185;

M. Czakon, J. Gluza and T. Riemann, The Planar four-point master integrals for massive two-loop Bhabha scattering, Nucl. Phys. B 751 (2006) 1.

[9] S. Jadach, W. Placzek and B.F.L. Ward, BHWIDE 1.00: $O(\alpha)$ YFS exponentiated Monte Carlo for Bhabha scattering at wide angles for LEP1/SLC and LEP2, Phys. Lett. B 390 (1997) 298.

[10] S. Agostinelli et al., Geant4 - a simulation toolkit, Nucl. Instrum. Meth. A 506 (2003) 250.

[11] MOKKA, A simulation program for linear collider detectors, http://polzope.in2p3.fr:8081/MOKKA/.

[12] T.C. Awes, F.E. Obenshain, F. Plasil, S. Saini, S.P. Sorensen and G.R. Young, A simple method of shower localization and identification in laterally segmented calorimeters,

Nucl. Instrum. Meth. 311 (1992) 130.

[13] I. Sadeh, Luminosity measurement at the International Linear Collider, arXiv: 1010.5992.

[14] H. Abramowicz et al., Redefinition of the geometry of the luminosity calorimeter, EUDET-Memo-2008-09 (2008), http://www.eudet.org.

[15] H. Abramowicz et al., Revised requirements on the readout of the luminosity calorimeter. EUDET-Memo-2008-08 (2008), http://www.eudet.org.

[16] J. Brau et al., ILC Reference Design Report, arXiv:0712.1950.

[17] D. Schulte, Beam-beam simulations with guinea-pig, CERN-PS-99-014LPCLIC-Note 387 (1998).

[18] A. Seryi, T. Maruyama and B. Parker, IR optimization and anti-DID, SLAC-PUB-11662 (2006). 
[19] P. Bambade, V. Drugakov and W. Lohmann, The impact of Beamcal performance at different ILC beam parameters and crossing angles on stau searches, Pramana J. Phys. 69 (2007) 1123.

[20] A. Heikkinen and N. Stepanov, Bertini Intra-nuclear Cascade implementation in Geant4, Proceedings of CHEP03 (2003) La Jolla, California [nucl-th/0306008].

[21] C. Coca et al., Expected electromagnetic and neutron doses for the BeamCal at ILD Rom. J.Phys 55 (2010) 687.

[22] T. Tauchi and K. Yokoya, Nanometer beam-size measurement during collisions at linear colliders, Phys. Rev. E 51 (1995) 6119.

[23] T. Tauchi, K. Yokoya and P. Chen, Pair creation from beam-beam interaction in linear colliders, Part. Accel. 41 (1993) 29.

[24] K. Ito, Study of Beam Profile Measurement at Interaction Point in International Linear Collider, arXiv:0901.4151.

[25] A. Elagin, The optimized sensor segmentation for the very forward calorimeter, in proceedings of the 2005 International Linear Collider Physics and Detector Workshop, Snowmass, Colorado, ECONF C0508141 (2005) ALCPG0719.

[26] J. Blocki et al., LumiCal new mechanical structure, EUDET-Memo-2009-10 (2009), http://www.eudet.org/.

[27] J. Blocki et al., Laser alignment system for LumiCal, EUDET-Report-2008-05 (2008), http://www.eudet.org.

[28] C. Rimbault, P. Bambade, K. Monig and D. Schulte, Impact of beam-beam effects on precision luminosity measurements at the ILC, 2007 JINST 2 P09001.

[29] W. Kilian, WHIZARD: A generic Monte-Carlo integration and event generation package for multi-particle processes, LC-TOOL-2001-039 (2001).

[30] V.N. Pozdnyakov, Two-photon interactions at LEP, Phys. Part. Nucl. Lett. 4 (2007) 289.

[31] S. Jadach, E. Richter-Was, B.F.L. Ward and Z. Was, Monte Carlo program BHLUMI for Bhabha scattering at low polar angle with Yennie-Frautschi-Suura exponentiation, Comp. Phys. Commun. 70 (1992) 305.

[32] B. Pawlik et al., BARBIE V4.3, Simulation-package of the LumiCal Detector, http://www.ifj.edu.pl/dept/no1/nz13/barbi.php.

[33] I. Smiljanic et al., Towards a final selection for luminosity measurement, Proceedings of the Workshop of the Collaboration on Forward Calorimetry at ILC (2008), Belgrade Serbia.

[34] Ch. Grah et al., Report to the Detector R\&D Panel - Instrumentation of the Very Forward Region, Hamburg, Germany (2007), http://www.desy.de/prc/docs_rd/prc_rd_02_01_update_05_07.pdf.

[35] S. Boogert et al., Polarimeters and Energy Spectrometers for the ILC Beam Delivery System, 2009 JINST 4 P10015.

[36] S-DALINAC: Superconducting DArmstadt LInear ACcelerator, http://www.ikp.tu-darmstadt.de/beschleuniger_1/S-DALINAC.de.jsp.

[37] Ch. Grah et al., Radiation hard sensor for the BeamCal of the ILD detector, Proceedings of the IEEE conference, October 27 - November 3 (2007) Honolulu, U.S.A.

[38] Ch. Grah et al., Polycrystalline CVD Diamonds for the Beam Calorimeter of the ILC, IEEE Trans. Nucl. Sci. 56 (2009) 462. 
[39] J. Blocki et al., Silicon Sensors Prototype for LumiCal Calorimeter, EUDET-Memo-2009-07 (2009), http://www.eudet.org.

[40] M. Idzik et al., Status of VFCAL, EUDET-memo-2008-01 (2008), http://www.eudet.org.

[41] E. Beuville et al.. AMPLEX, a low-noise, low-power analog CMOS signal processor for multi-element silicon particle detectors, Nucl. Instrum. Meth. A 288 (1990) 157.

[42] M. Idzik, Sz. Kulis and D. Przyborowski, Development of front-end electronics for the luminosity detector at ILC, Nucl. Instrum. Meth. A 608 (2009) 169.

[43] http://www.home.agilent.com.

[44] M. Idzik, K. Swientek and Sz. Kulis, Development of a Pipeline ADC for the Luminosity Detector at ILC, 2010 JINST 5 P04006.

[45] IEEE standard for terminology and test methods for analog-to-digital converters, IEEE-STD-1241 (2000).

[46] J.L. McCreary and P.R. Gray, All-MOS charge redistribution analog-to-digital conversion techniques. I, IEEE J. Solid-State Circ. 10 (1975) 371.

[47] H. Spieler, Semiconductor Detector Systems. Oxford University Press, Oxford U.K. (2005).

[48] R. Gregorian, K.W. Martin and G.C. Temes, Switched-capacitor circuit design, IEEE Proc. 71 (1983) 941.

[49] F. Silveira, D. Flandre and P.G.A. Jespers, $A g_{m} / I_{D}$ based methodology for the design of CMOS analog circuits and its application to the synthesis of a silicon-on-insulator micropower OTA, IEEE J. Solid-State Cir. 31 (1996) 1314.

[50] S. Rabii, Design of Low-Voltage Low-Power Sigma-Delta Modulators, PhD thesis, Stanford University (1998).

[51] Y. Arai, Electronics and sensor study with the OKI SOI process, in proceedings of the Topical workshop on electronics for particle physics (TWEPP-07), September 3-7 (2007) Prague, Czech Republic. 